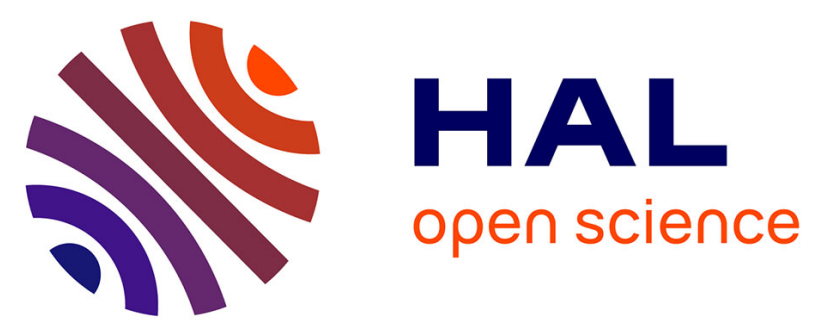

\title{
On the Impact of Linear Siloxanated Side-chains on the Molecular Self-Assembling and Charge Transport Properties of Conjugated Polymers
}

Narayanaswamy Kamatham, Olzhas A Ibraikulov, Pablo Durand, Jing Wang, Olivier Boyron, Benoît Heinrich, Thomas Heiser, Patrick Lévêque, Nicolas Leclerc, Stéphane Méry

\section{To cite this version:}

Narayanaswamy Kamatham, Olzhas A Ibraikulov, Pablo Durand, Jing Wang, Olivier Boyron, et al.. On the Impact of Linear Siloxanated Side-chains on the Molecular Self-Assembling and Charge Transport Properties of Conjugated Polymers. Advanced Functional Materials, 2020, pp.2007734. 10.1002/adfm.202007734 . hal-03006159

\section{HAL Id: hal-03006159 https://hal.science/hal-03006159}

Submitted on 15 Nov 2020

HAL is a multi-disciplinary open access archive for the deposit and dissemination of scientific research documents, whether they are published or not. The documents may come from teaching and research institutions in France or abroad, or from public or private research centers.
L'archive ouverte pluridisciplinaire HAL, est destinée au dépôt et à la diffusion de documents scientifiques de niveau recherche, publiés ou non, émanant des établissements d'enseignement et de recherche français ou étrangers, des laboratoires publics ou privés. 


\section{On the Impact of Linear Siloxanated Side-chains on the Molecular Self-Assembling and Charge Transport Properties of Conjugated Polymers}

Narayanaswamy Kamatham, ${ }^{1 \ddagger}$ Olzhas A. Ibraikulov, ${ }^{2 \ddagger}$ Pablo Durand, ${ }^{3}$ Jing Wang, ${ }^{2}$ Olivier Boyron, ${ }^{4}$ Benoît Heinrich, ${ }^{1}$ Thomas Heiser, ${ }^{2}$ Patrick Lévêque,${ }^{2}$ Nicolas Leclerc ${ }^{3}$ and Stéphane Méry $^{1 *}$

${ }^{1}$ Drs. N. Kamatham, B. Heinrich and S. Méry, Institut de Physique et de Chimie des Matériaux de Strasbourg (IPCMS), CNRS, Université de Strasbourg, UMR 7504, 23 rue du Loess, 67034, Strasbourg, France. Email: mery@ipcms.unistra.fr.

${ }^{2}$ Dr. O. A. Ibraikulov, Jing Wang, Dr. P. Lévêque and Pr. T. Heiser, Laboratoire ICube, CNRS, Université de Strasbourg, UMR 7357, 23 rue du Loess, 67037, Strasbourg, France.

${ }^{3}$ P. Durand and Dr. N. Leclerc, Institut de Chimie et Procédés pour l'Energie, l'Environnement et la Santé (ICPEES), CNRS, Université de Strasbourg, ECPM, UMR 7515, 25 rue Becquerel, 67087, Strasbourg, France.

${ }^{4}$ O. Boyron, Laboratoire de Chimie Catalyse Polymères et Procédés (C2P2), Université de Lyon, CPE Lyon, CNRS, UMR 5265, 43 Bd du 11 Novembre 1918, 69616, Villeurbanne, France.

$\$$ These authors contributed equally to this work

Keywords. Siloxane, oligodimethylsiloxane, siloxane side-chain, side-chain polymer, semiconducting polymer, charge-carrier mobility, mesomorphic polymer 


\begin{abstract}
Herein is reported the impact of the functionalization of four different semiconducting polymer structures by a linear siloxane-terminated side-chains. The latter was tetrasiloxane $\left(\mathbf{S i}_{4}\right)$ or trisiloxane (Si3) chains, substituted at their extremity to a pentylene linker. The polymer structure was based on 5,6-difluorobenzothiadiazole comonomer (PF2), a diketopyrrolopyrrole unit (PDPP-TT), a naphtalediimide unit (PNDI-T2) and a poly[bis(thiophen-2yl)thieno[3,2,b]thiophene (PBTTT). The properties of these siloxane-functionalized polymers were scrutinized and compared with the ones of their alkyl-substituted polymer analogues. The impact of the alkyl-to-siloxane chain substitution clearly depends on the molecular section of the side-chains. When a branched 2-octyldodecyl chain $\left(\mathbf{C}_{20}\right)$ was replaced by a $\mathbf{S i}_{4}$ chain of same molecular section, the greatest impact was the strong increase of the $\pi$-stacking overlap of the polymer backbones. This effect led to a significative enhancement of the charge mobility values of the polymers. As in-plane and out-of-plane mobility were increased simultaneously, this $\pi$-overlap enhancement effect happens to be preponderant over the polymer orientation variations. When a linear tetradecyl chain $\left(\mathbf{C}_{14}\right)$ was replaced by a linear $\mathbf{S i} 3$ chain of twice larger molecular section, the polymer structure was profoundly affected. While PBTTT-C 14 is crystalline and purely edge-on, PBTTT-Siz is mesomorphic and shows a mixed face-on/edgeon orientation.
\end{abstract}




\section{Introduction}

In the state-of-the-art design of semiconducting $\pi$-conjugated polymers, side-chain engineering constitutes one of the key aspects.[1] Side-chains are naturally needed for providing a satisfactory solubility required for purification and device processing. They also strongly impact the molecular ordering and thin-film morphology, which ultimately determine device performances. Alkyl side-chains have been investigated for a long time, and the accumulated knowledge has become today essential for the design of high-performing polymers used in electronics, sensors and display technologies, in particular.[1a,2] Thus, an abundant literature has been produced reporting the important role of the location, length and branching position of alkyl side-chains on the polymer solubility, backbone conformation and polymer structure.[1,2]

Alternatively, recent years have witnessed the development of new types of side-chains containing different functionalities or functional groups.[1a] Actually, this research field has rapidly emerged to meet the demand i) to ease the solubilization of highly $\pi$-stacked polymer systems, [3] ii) to bring new solubility properties to the materials in order to substitute classically used harmful halogenated solvents towards eco-friendly ones, [4] or iii) to provide the materials with special affinity of reactive groups for the targeted devices (as interfacial layers, [5] for layer immobilization[6]...) or applications (in sensing,[7] bioelectronics,[8] thermoelectricity[8]...). Clearly, all modifications brought in the nature of the side-chain not only affect the polymer's solubility but also a broad range of its physical parameters. Such a global impact makes difficult the rationalization of the functional side-chain substitution, but this remains essential for the design of advanced polymers, such as for efficient electronic applications. For instance, oligo(ethyleneoxide) (EO) side-chain have been used to provide polymers with a hydrophilic character to improve their solubility in water or polar solvent,[9] and for a purpose of bioelectronic applications,[10] in particular. The replacement of alkyl by EO side-chains was found to drastically affect many physical parameters of the polymers, such as the $\pi$-stacking distances and the energy levels of the polymers, and consequently the polymer's performance in field effect transistor (OFET) devices.[11] A fine tuning of the EO chains substitution could also be used to adjust the nanophase segregation in an all-polymer organic photovoltaic (OPV) device.[12] Fluoroalkyls have also been used as side-chains for its fluorophilic character, which resulted in a strong lamellar self-organization with the formation of well-defined microstructures of high mobility.[13] Alternatively, the fluorophobic effect was found to 
reduce the miscibility between compounds in mixtures, which facilitated the fabrication of welldefined bilayer systems for ambipolar OFETs,[14] but conversely, aggravated the phase segregation with PCBM in bulk heterojunction (BHJ) solar cells.[15]

(Dimethyl)siloxane segments have also been inserted in side chains of $\pi$-conjugated polymers. Mei et al. first introduced a trisiloxane-terminated side chain (branched pendant through an alkyl linker) in isoindigo-based polymers.[16] As compared to a branched (2-octyldodecyl) alkyl chain with a proximal cumbersome ramification, the authors reported a closer $\pi$-stacking distance and larger coherence length, ultimately leading to higher OFET mobility values. Later on, other authors confirmed in different polymer structures, that hybrid siloxane side chains allowed the formation of closely $\pi$-stacked polymers into large fibrillar structures, leading to OFET mobilities over $1 \mathrm{~cm}^{2} /$ V.s.[17,18] While opposite results were published regarding solubility, it seems that polymers with trisiloxane (laterally attached) hybrid side chains exhibit a lower solubility than the one substituted with branched alkyl chains.[17,19-21] It is only with long linear oligosiloxane chains (average of 10 siloxane units per chain) that a better solubility was measured by Ohnishi et al.[22] The substitution of alkyl to siloxane hybrid side-chains was found to favor the formation of face-on polymer orientation, [16,17,21,23,24] although the nature of the solvent seemed to be determinant.[25,26] Indeed, face-on polymer orientation is highly favorable for OPV application, however the siloxane functionalization needs to be finely tuned in order to control the donor-acceptor nanophase separation.[21-24,27] Such a controlled siloxane functionalization favorably offered promising photovoltaic devices with efficiencies over $10 \%$ with subtly mixed alkyl/siloxane side-chains polymer systems.[19,28-31]

The results obtained so far on siloxane functionalization are very promising for the development of high performing $\pi$-conjugated polymers for electronic applications, indeed. So far, however, the published studies are essentially based on a single type of siloxane chain, i.e. a branched heptamethyltrisiloxane, which is connected to alkyl linkers by the central silicon atom. The unique variable parameter was the length of the alkyl linker.[32,33] Only two reports have been published by considering a different siloxane side-chain configuration based of an endattached siloxane segment. The first one is a linear polydispersed oligosiloxane side-chain reported by Onishi et al.[22], and the second is a linear hepta(dimethylsiloxane) segment that was published by Y. Ding et al.[34] during this manuscript preparation. Thus, there is clearly a need to broaden the scope of the study to linear siloxane side-chains functionalization. 
Moreover, such linear siloxane-terminated chain opens up the valuable variation study of the siloxane chain length.

Herein we report, the functionalization of $\pi$-conjugated polymers by well-defined linear siloxane-terminated side-chains. The functionalization has been performed on a series of four different polymer structures, which can be considered as state-of-the-art polymers for their optoelectronic properties and more specifically for their excellent charge transport properties: a diketopyrrolopyrole-based copolymer (PDPP-TT),[35] a 5,6-difluorobenzothiadiazole-based copolymer (PF2),[36,37] a nathtalenediimide-based polymer (PNDI-T2)[38] and a poly[bis(thiophen-2-yl)thieno[3,2-b]thiophene] (PBTTT).[39] Two types of linear siloxane chains have been considered: a trisiloxane ( $\left.\mathrm{Si}_{3}\right)$ and a tetrasiloxane $\left(\mathrm{Si}_{4}\right)$ chain, connected to the polymer backbone via a pentylene linker. The study clearly aims at rationalizing the role of the substitution of classical alkyl side-chains to hybrid linear siloxane side-chains on various properties of the polymers, namely the solubility, energy levels, structure, orientation, and finally, on charge transport properties. The outcome of this study will help drawing the general trends in hybrid linear siloxane-terminated side-chain functionalization and more generally, it will provide insights in the design of siloxane chain-containing polymers for electronics.

\section{Results and discussion}

\subsection{General consideration on dimethylsiloxane chains}

Before investigating the impact of the linear hybrid siloxane chain substitution on the physical properties of the polymer semiconductors, it appears essential to report the main physicochemical properties of dimethylsiloxane chains (-SiMe $-\mathrm{O}-)$ and their distinctive features as compared to their carbon-based counterparts.[40-43]

As a first general comment, oligodimethylsiloxanes show several characteristics that make them valuable for their integration into organic materials, such as ease of fabrication, transparency to UV-visible regions, high thermal stability, low electrical conductivity, biocompatibility and high fluidity.

To begin, oligodimethylsiloxanes are hydrophobic and lipophilic. For example, polydimethylsiloxane (PDMS) films grafted onto silicone wafer exhibit contact angles around $115^{\circ}$.[40] The high hydrophobicity of siloxanes was found to be responsible for the enhanced 
stability observed in siloxane-functionalized materials used in organic electronics, in particular.[17,44] Regarding solubility and as a first approximation, the solubility of oligodimethylsiloxanes is comparable to the ones of alkanes of similar length. Thus, PDMS exhibits a very low solubility parameter $\delta(14.9-15.6 \mathrm{MPa}[40])$ that is close to the one of liquid paraffins (16-16.5 $\mathrm{MPa}[45])$. Nevertheless, the presence of weak dipole moments in the backbone make PDMS soluble and miscible in a larger variety of solvents, having a non-polar or a weakly polar nature.[40,46] This peculiar feature arises from the extreme flexibility of the siloxane backbone and pending methyl groups that can readily adjust their conformation (by masking or uncovering the "polar" oxygen atoms of the backbone) to suit their surrounding environment.[47]

As mentioned above, dimethylsiloxane chains exhibit a remarkable flexibility, which is probably the most striking feature of silicone materials. This flexibility far exceeds what can be found for any alkyl or ether chains, and arises from the peculiar nature of the $\mathrm{Si}-\mathrm{O}$ bond.[48] First, the Si-O bond length $(1.63 \AA)$ is significantly higher than the C-C (1.53 $⿱$ A) and C-O (1.41 $\AA$ ) bonds.[49] Second, the oxygen skeletal atoms are small and unencumbered by side-groups. Third, the partial double bond character between the silicon and oxygen atoms (due to $\mathrm{p}_{\pi}-\mathrm{d}_{\pi}$ interaction) results in a large $\mathrm{Si}-\mathrm{O}-\mathrm{Si}$ angle $\left(\approx 150^{\circ}\right)$, as compared to the usual tetrahedral value $\left(\approx 110^{\circ}\right)$ of C-C-C or C-O-C angles.[48,49] These concomitant effects lead to a drastic reduction of the energy barrier for the rotation around the Si-O bond and for the rotation of the methyl groups attached to the silicon atoms. Then, bond angles can easily pass through $180^{\circ}$ and methyl groups can flip almost freely.[48] As a result, PDMS stands among the most flexible polymers and exhibits a glass transition temperature $(T \mathrm{~g})$ as low as $-120^{\circ} \mathrm{C}$.[50] PDMS also hardly crystallizes at very low temperature to give a melting point $(\mathrm{mp})$ at $-43^{\circ} \mathrm{C},[50]$ when polyethylene and poly(ethyleneoxide) present a melting usually above 110 and $65^{\circ} \mathrm{C}$, respectively.[51] The great difficulty of PDMS to crystallize also arises from the irregularity of the cross-section along the chains (due to the succession of $-\mathrm{Me}_{2} \mathrm{Si}$ - and $-\mathrm{O}$ - units of very different volume), that obstructs their efficient packing.[52] The extreme dynamic mobility of the dimethylsiloxane chains are also responsible in large part, for many other singular physical properties such as small change in viscosity with temperature, low cohesion energy density, low surface tension $\left(21 \mathrm{mN} . \mathrm{m}^{-1}\right)$, low dielectric constant and high gas permeability.[40,43]

Segregation constitutes another major feature of dimethylsiloxanes. Actually, siloxanes show a strong incompatibility with other chemical species, that expresses through their strong affinity 
with chains of same nature.[53,54] This characteristic arises, to a first approximation, to their very low solubility parameter $\delta$. This effect is not as strong as fluorophobic effect of perfluoroalkyl chains though, but it is powerful enough to drive heavy nanosegregation. This phenomenon has widely been exploited in block-copolymer systems as well as in liquid crystals, for which small ratios of siloxane chains were able to generate a wide variety of nanostructures and mesomorphic structural organizations.[55-58]

Geometrical consideration of dimethylsiloxane chains is the last information of interest to report here. In a segregated material, the structural organization is naturally controlled by the difference of molecular areas and volumes of the antagonistic moieties. Thus, the difference of molecular areas mainly determines the type of organization, while volumes mainly affect the molecular distances. Then, the cross-sectional areas $\sigma$ at room temperature of a dimethylsiloxane chain $\left(\sigma=41 \AA^{2}\right)$ is about twice larger as compared to a linear alkyl or ether chains ( $\sigma=21-22 \AA^{2}$ ) (see supporting information). That is to say that a linear dimethylsiloxane chain has a molecular section comparable to the one of two linear alkyl chains or else, of one ramified alkyl chain, as illustrated in figure 1. The molecular volume $v$ of a dimethylsiloxane segment at room temperature $\left(v=127 \AA^{3}\right)$ is also significantly larger than the one of a $-\mathrm{CH}_{2}$ chain $\left(v=27 \AA^{3}\right)$, meaning - $\mathrm{SiMe}_{2} \mathrm{O}$ - occupies the same volume than a pentylene $-\mathrm{C}_{5} \mathrm{H}_{10}$ - chain (see ESI).
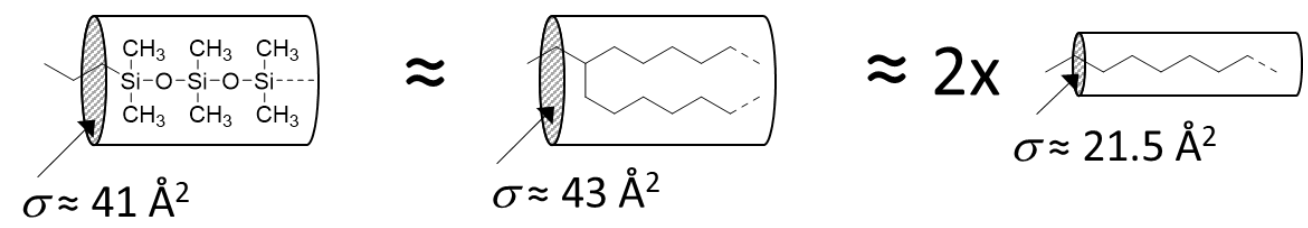

Figure 1. Comparison of the cross-section area $(\sigma)$ at room temperature of a linear oligo(dimethylsiloxane) chain with the one of a linear and a ramified alkyl chain.

To summarize the characteristic features of siloxane chains, a chart is reported below to highlight the main differences of oligodimethylsiloxanes with the ones of high molecular weight $n$-alkanes (paraffin wax). These differences should naturally have an impact on the properties of our semiconducting polymers. An influence of the alkyl-to-siloxane chain substitution is thus to be expected in solubility, thermal properties, structural organization, and subsequently in charge transport properties, that will be extensively examined in the following. 
Chart. Main differences in the properties of oligodimethylsiloxanes with the ones of high molecular weight n-alkanes.

\section{Higher hydrophobicity}

Miscible in a broader range of solvents

Higher flexibility (room-temperature liquids with $\mathrm{Tg}<-120^{\circ} \mathrm{C}$ )

Higher propensity for microphase separation

\section{Twice larger molecular cross-section}

\subsection{Molecular design and synthesis}

The four different investigated polymer structures are depicted in figure 2. Two different couples of (alkyl/siloxane) side-chains have been studied, depending on the polymer structure. For the three first polymers (PF2, PDPP-TT and PNDI-T2), whose best semiconducting properties are achieved with long ramified alkyl chains, $[35,36,38,39]$ the comparison was made by using 2-octyldodecyl $\left(\mathbf{C}_{\mathbf{2 0}}\right)$ chain and a linear tetrasiloxane $\left(\mathbf{S i}_{4}\right)$ chain, namely 1-n-butyl1,1,3,3,5,5,7,7-octamethyltetrasiloxane. For the last polymer (PBTTT), whose highest charge transport mobilities are obtained with linear alkyl chains,[39] it was synthetized with $n$ tetradecyl $\left(\mathbf{C}_{14}\right)$ chain and a short linear trisiloxane $\left(\mathbf{S i}_{3}\right)$ chain, i.e. 1,1,1,3,3,5,5heptamethyltrisiloxane. For all siloxane chain substitutions, the siloxane segment is connected to the conjugated monomer by its extremity, via a short pentylene linker (figure 2).

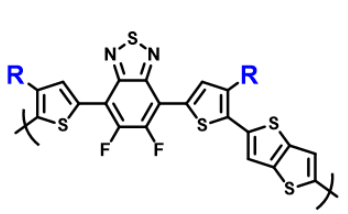

PF2

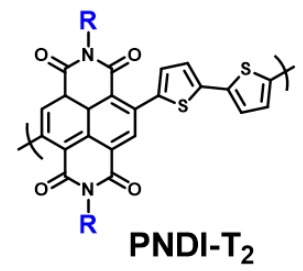

PNDI-T 2

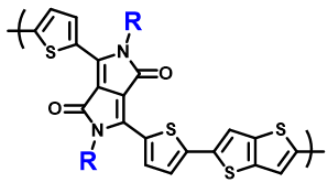

PDPP-TT

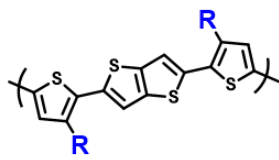

PBTTT

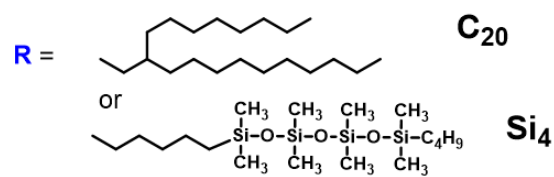

$\mathbf{R}=$

or

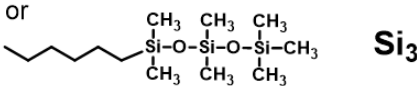

Figure 2. Structure of the four polymers series substituted with alkyl and siloxane chains. 
The synthesis of the alkyl-substituted polymers PF2-C20,[36] PNDI-T2-C20,[38] PDPP-TT$\mathbf{C}_{20}[35]$ and PBTTT-C ${ }_{14}[39]$ has already been reported. The same procedures were used to prepare their siloxane counterparts (see figure 3 and experimental details in ESI). The key step was the preparation of the dibromide siloxane-functionalized comonomers which were used for the last Stille cross-coupling polycondensation step. The siloxane comonomers were prepared by hydrosilylation of the corresponding $\omega$-pentenyl-substituted precursors with the trisiloxane or tetrasiloxane chain, by using the Karstedt catalyst. In the specific case of PNDI-T2-Si4, the siloxane-functionalized naphtalenediimide comonomer was obtained by direct imidation of the naphthalene tetracarboxylic dianhydride with $\omega$-tetrasiloxane pentyl-1-amine. The later was obtained from the cleavage of the corresponding $\mathrm{N}$-substituted phtalimide in mild conditions, by using methylamine in ethanol (figure 3).[59] This procedure successfully avoided the use of basic and acid treatment conditions that are detrimental to siloxanes (hydrolysis). While the trisiloxane chain was commercially available, the tetrasiloxane chain ( $\mathrm{Si} 4-\mathrm{H})$ was prepared in one pot, by ring opening of cyclohexamethyltrisiloxane with $n$-Buli, followed by the addition of chlorodimethylsilane.[60]

The final polymers were characterized by size exclusion chromatography (SEC) in 1,2,4trichlorobenzene at $150^{\circ} \mathrm{C}$ (see plots in figure S2 and data in Table 1) and by elemental analysis. The PBTTT polymer series which are soluble in chloroform were also characterized by NMR. The characterization data are in good agreement with the targeted polymers (see ESI). It is worth to mention that the SEC profiles show monomodal distributions with no trace of residual monomers. All polymers show $M n$ in a similar range, except PBTTT-C $\mathbf{1}_{\mathbf{1 4}}$ that exhibits a slightly lower molecular weight (Table 1). 


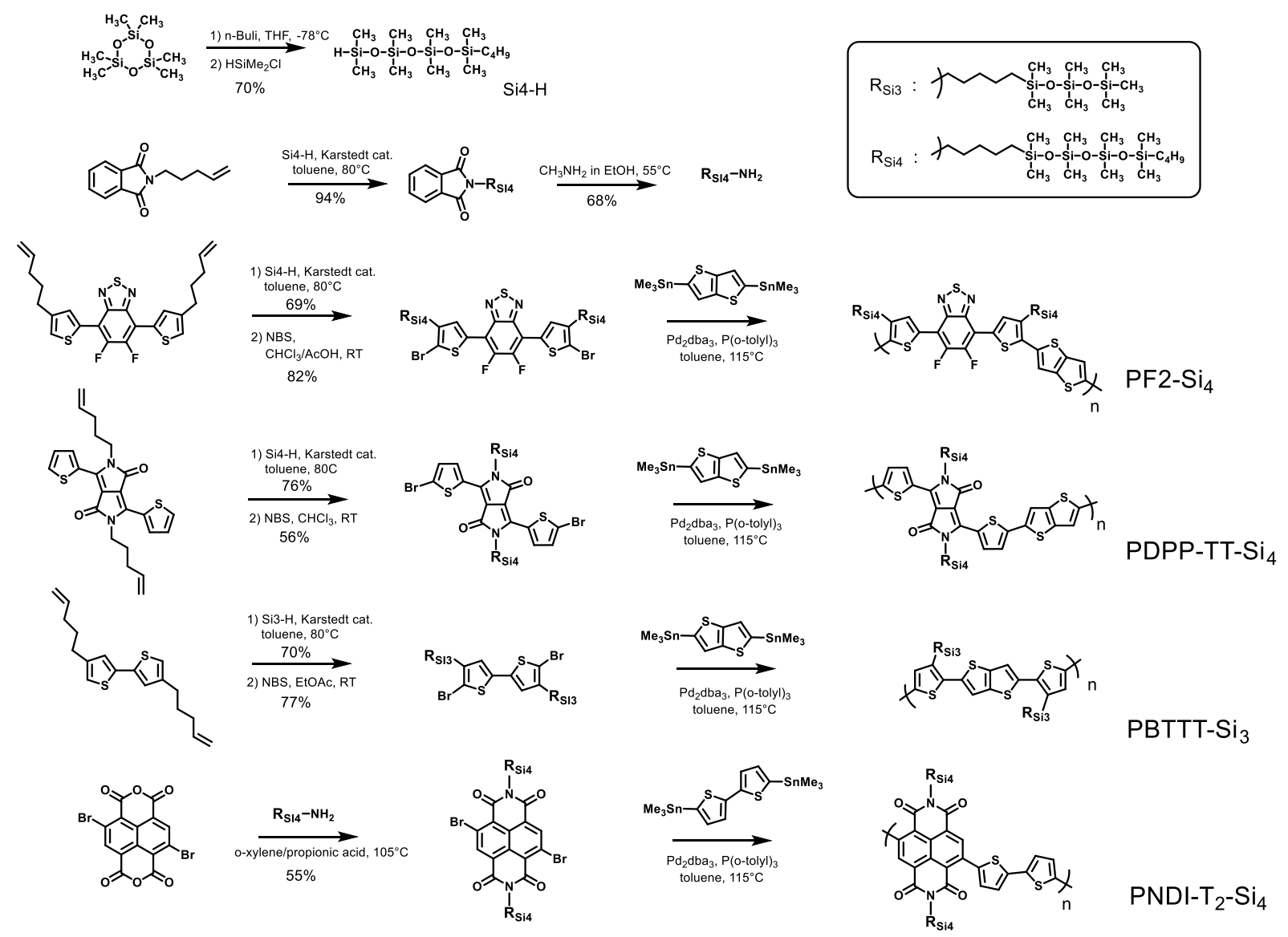

Figure 3. Synthesis of the materials

\subsection{Energy levels and absorption}

In order to estimate the energy levels of the polymers, cyclic voltammetry (CV) and photoelectron spectroscopy in air (PESA) on thin films have been performed (see figures S3 and $\mathrm{S} 4$, respectively). In $\mathrm{CV}$, all potentials are referred to the saturated calomel electrode that was calibrated at $0.38 \mathrm{~V}$ versus the $\mathrm{Fc} / \mathrm{Fc}^{+}$system.[61] The voltammograms for the electron donor polymers only showed oxidation potentials, while reduction waves could only be observed for the electron acceptor PNDI-T2 2 series. In Table 1 are given the data of all measurements, including the calculated HOMO and LUMO values. The HOMO values obtained by CV and PESA are in good agreement. For each polymer series, a clear trend can be observed for the HOMO-LUMO values depending on the chain substitution. When substituting the alkyl chain by the linear hybrid siloxane side-chains, the calculated energy levels are slightly destabilized. This observation is in good agreement with previous studies showing a similar upward trend in HOMO value after alkyl to hybrid siloxane side-chain substitution.[16,21,24,62] 
The polymer absorption spectra recorded in solution and in thin films are reported in figures S5 (ESI) and in figure 4, respectively. Absorptions in solution were performed in orthodichlorobenzene $(o \mathrm{DCB})$ at temperatures ranging from 25 to $95{ }^{\circ} \mathrm{C}$. These analyses allowed to investigate the side-chain impact on polymer solubility properties and provided also an early stage of information regarding the structural organization of the polymers.

To start with the alkylated PF2 polymer (PF2-C20), a clear transition between two different states can be observed,[36] i.e. a soluble state at high temperature (above $85^{\circ} \mathrm{C}$ ) and an aggregated state at lower temperature (see figures S5). The latter exhibits a two bands spectrum, as commonly observed in D-A polymers.[63] The highest wavelength band is generally attributed to the intramolecular charge transfer (ICT) between electron rich and electron deficient units within the conjugated backbone, while the band at highest energy can be attributed to a $\pi-\pi^{*}$ transition. PF2-C20 exhibits an additional feature related to its solid-state structuration, that is to say a split of the highest wavelength (ICT) band into two peaks, usually referred as the $0-0$ band (lowest energy, centered on $700 \mathrm{~nm}$ ) and 0-1 band (highest energy, centered on $640 \mathrm{~nm}$ ).[36] The 0-0 band is associated to the segregated stacking of polymer chains on each other's, while the $0-1$ band is related to a lowest ordered structuration (also called mixed stacking, see figure 5).[64] Under heating, the ICT vibronic band disappears progressively to give a new broad featureless band, strongly blue shifted and centered at around $550 \mathrm{~nm}$, characteristic of a well-soluble polymer state. In the meantime, the highest energy $\pi$ $\pi^{*}$ band is also blue shifted from 450 down to $390 \mathrm{~nm}$. The scenario is significantly different for the hybrid siloxane-based PF2 (PF2-Si4). At low temperature indeed, PF2-Si4 naturally exhibits similar features as for PF2-C20 with the presence of a $\pi-\pi^{*}$ and a vibronic ICT band. However, the shape of this vibronic ICT band is different in PF2-Si4, and the absorption ratio between the 0-1 and 0-0 bands evolve differently by heating. More importantly and unlike PF2$\mathbf{C}_{20}$, the soluble state behavior is never reached in PF2-Si4, even after heating at $95^{\circ} \mathrm{C}$. This observation indicates a lower solubility of PF2-Si4 in $o$ DCB, as compared to PF2-C20. It is confirmed by additional Hansen solubility parameter measurements done for both polymers (figure S6 and Table S6). The latter reveal a significant shift in position of the solubility spheres, leading to a slightly larger relative energy difference (RED) of $o$ DCB (and thus a lower solubility) for PF2-Si4, in comparison to PF2-C20. On the other hand, the radius of the solubility spheres is significantly larger for $\mathbf{P F}_{2}-\mathbf{S i}_{4}$ (4.9 compared to 4.0 for $\mathbf{P F}-\mathbf{C}_{20}$ ), indicating a broader range of potential solvents for $\mathbf{P F}_{2}-\mathbf{S i}_{4}$.

For PDPP-TT polymer series, a completely different behavior is observed: both alkyl and siloxane chain substitution lead to a quite similar absorption spectra in solution (figures S5). 
The polymers essentially show a broad band at around $830 \mathrm{~nm}$, which slightly drift hypsochromically by heating. The two PDPP-TT polymers thus show a similar good solubility in $o \mathrm{DCB}$ at room temperature.

For the PNDI-T2 polymers, the behavior looks like the ones of PF2 series but the effect of the side-chain substitution is even more pronounced. Although, both polymers are aggregated at room temperature, their absorption spectra in $o$ DCB exhibit a quite different shape, essentially regarding their ICT bands (figures S5). Actually, PNDI-T2-C20 presents at $25^{\circ} \mathrm{C}$ a rather featureless ICT band, in which two peaks can nevertheless be distinguished, at ca 650 and 710 nm. At same conditions PNDI-T2-Si4 exhibits a highly structured ICT band with three different vibronic peaks, at approximately 630, 720 and $810 \mathrm{~nm}$. The PNDI-T2-Si4 ICT band is thus showing one additional band at low energy, thus expanding the bathochromic absorption over $100 \mathrm{~nm}$ (up to $900 \mathrm{~nm}$ ). As similarly discussed for PF2 polymers, it has been clearly shown that, using specific process conditions, PNDI-T2- $\mathbf{C}_{2}$ polymer could adopt two different stacking configurations, mixed and segregated, also called as form 1 and form 2, respectively (figures 5). The former was characterized by the emergence of a low-energy band, at around $810 \mathrm{~nm}$.[65] As a consequence, the much higher ordering observed in solution at $25^{\circ} \mathrm{C}$ for PNDI-T2-Si4, as compared to PNDI-T2-C20, strongly suggests a higher segregated stack polymer fraction (Form 1) in the siloxane-substituted PNDI-T2-Si4 polymer. Under heating moreover, the absorption spectra of the two polymers are also found to evolve differently. For PNDI-T2- $\mathbf{C}_{20}$, the initial ICT band rapidly disappears (above $75^{\circ} \mathrm{C}$ ) to exhibit a new broad, featureless and blue-shifted band centered at around $600 \mathrm{~nm}$, characteristic of a soluble state. For PNDI-T2-Si4 at contrary, the vibronic ICT band only decreases in intensity without vanishing totally, in favor of the emergence of a new broad band at ca $620 \mathrm{~nm}$. Although PNDI$\mathbf{T}_{2}$-Si4 gains in solubility by heating, it remains irremediably aggregated at high temperature, yet.

Finally, the PBTTT series reveals a different behavior as the previous ones. The replacement of a linear alkyl $\left(\mathbf{C}_{\mathbf{1 4}}\right)$ chain by a linear (though bulky) linear siloxane ( $\mathbf{S i}$ ) chain, leads to different absorption properties at low temperatures (figures S5). The room-temperature PBTTT-C 14 absorption spectrum exhibits a broad band centered at $485 \mathrm{~nm}$, including a shoulder at lower energies at around $600 \mathrm{~nm}$. This vibronic structure is a sign of crystallinity and already reflects an incomplete PBTTT-C14 dissolution.[66] Upon heating, this vibronic structure rapidly fades away to give a featureless broad band centered at around $475 \mathrm{~nm}$. Such a vibronic band is already absent at low temperature in the PBTTT-Si3 absorption, demonstrating either a higher solubility and/or a lower polymer chain ordering. This siloxane 
derivative only exhibits a broad band centered at $480 \mathrm{~nm}$, which remains almost unchanged by heating.

At this stage, a partial conclusion can already be drawn from the absorption in solution as a function of the side-chain substitution. Actually, two opposite behaviors can be noticed depending on whether the initial alkylated polymers are carrying branched alkyl chains or linear ones. When branched alkyl $\mathbf{C} 20$ chains are substituted by linear siloxane (Si4) chain (of similar molecular section, as illustrated in figure 1), the polymer shows a slightly reduced solubility in $o \mathrm{DCB}$, and seems to present a better solid-state ordering in its aggregated form. At the contrary for PBTTT, the replacement of the linear $\mathrm{C}_{14}$ chain by a bulkier $\mathrm{Si}_{3}$ chain leads to a significantly better solubility and/or else, a decrease of a polymer chain ordering.

Solid-state absorption can also provide valuable information regarding the impact of the alkylto-siloxane chain substitution on the solid-state polymer ordering (figure 4). To start with the PF2 series, both polymers exhibit similar thin film absorption profiles at first sight. However, a slightly higher 0-0/0-1 intensity ratio is observed for PF2-Si4, which suggests for the siloxanefunctionalized polymer a higher polymer ordering with a higher proportion of segregated stacked polymer chains. Along the PNDI-T2 2 series, the trend is even more pronounced. Indeed, if both polymers present similar UV-vis absorption profiles, PNDI-T2-Si4 exhibits a clear bathochromic shift with a very well defined vibronic structure, including a new peak at about $800 \mathrm{~nm}$. As previously discussed, this peak is strongly related to the existence of segregated stacks (form 1, see figure 5) in PNDI-T2-Si4, indicating a much higher ordering level, as

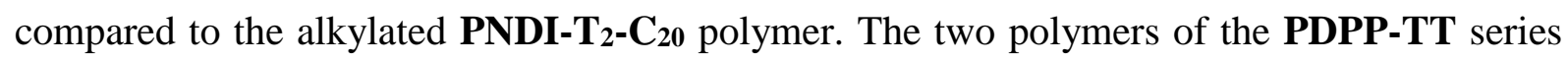
exhibit in solid-state, as in solution, a quite similar absorption spectrum. Finally, the PBTTT series exhibits a distinctive behavior. Indeed, PBTTT-C $\mathbf{1 4}_{\mathbf{1 4}}$ exhibits a standard UV-visible absorption spectrum (for as-cast polymer film) with a slight vibronic feature in the form of a shoulder at longer wavelengths. PBTTT-Siz shows a similar shoulder at higher wavelengths, but it shows more distinctly a new vibronic peak at high energy (at ca $480 \mathrm{~nm}$ ). The latter could likely be explained by the appearance of H-aggregates in PBTTT-Si3, arising from the bulkiness of the hybrid linear siloxane side-chain.

To sum up the thin film absorption results, all polymer series (with the exception of PDPP-TT) show some changes in the absorption spectrum as a function of the side-chain substitution, that reflects slight variations in the molecular packing. This latter aspect will be widely discussed in the following sections. At this stage however, the differences in the absorption spectra do not 
impact the polymer optical band gaps, as a similar absorption edge is reported within each polymer series.
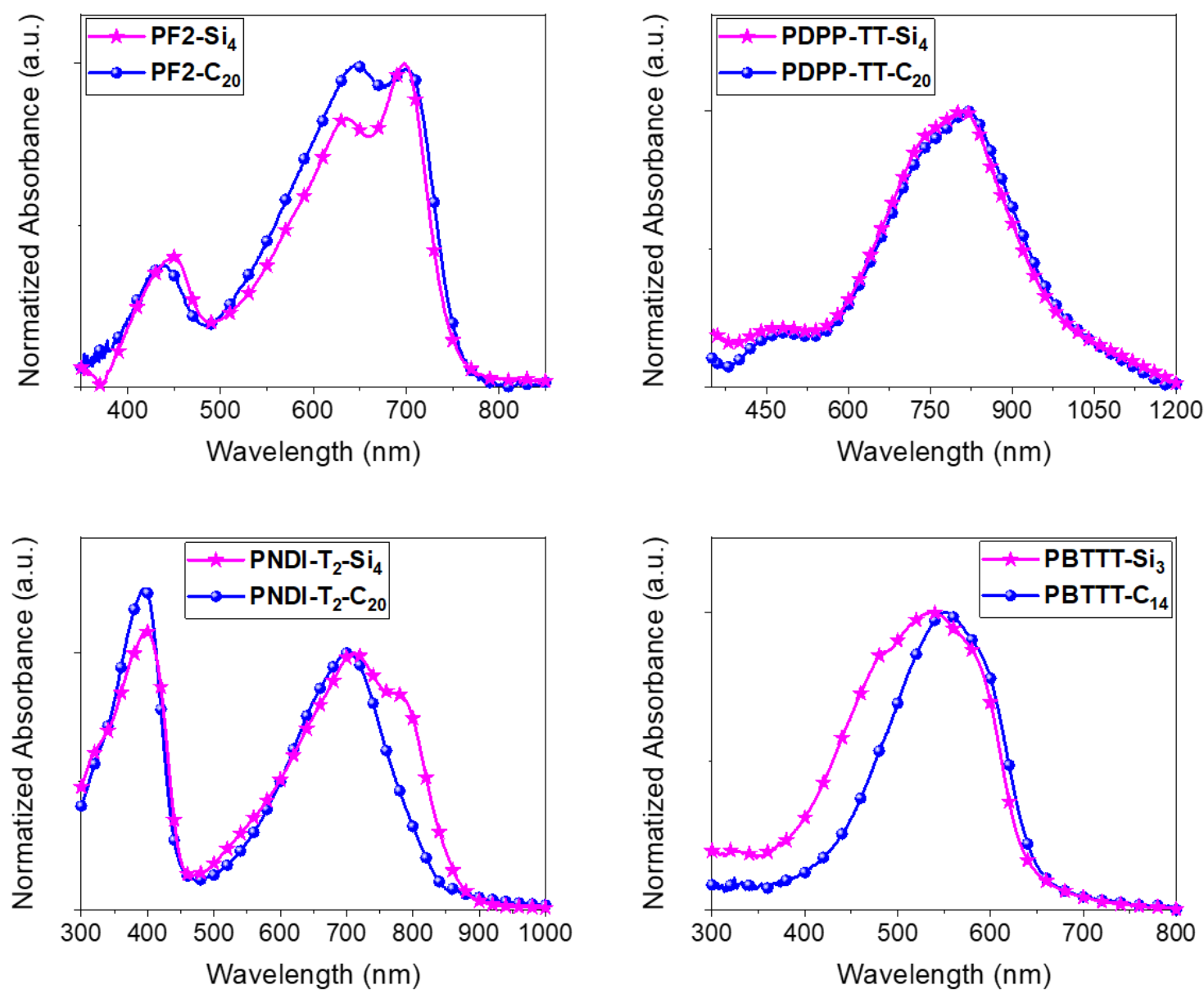

Figure 4. Comparison of the thin film absorption spectra of the four polymers structures as a function of their (alkyl versus siloxane) chain substitution.

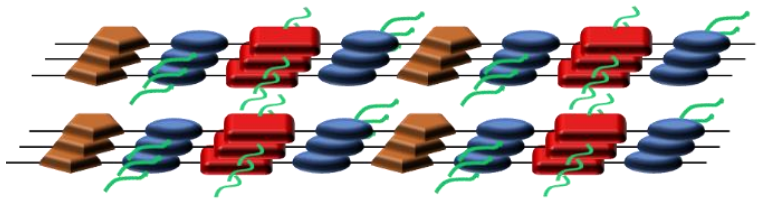

Segregated stacking

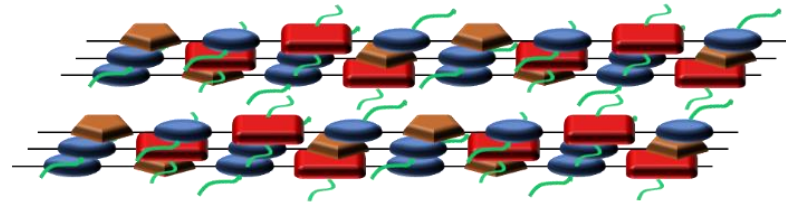

Mixed stacking

Figure 5. Schematic illustration of the segregated stacking (form 1) and of the mixed stacking (form 2) of lamellar organization of conjugated polymer backbones. In segregated stacking, the polymer backbones are piled up with a superimposition of same adjacent polymer sub-units, while in mixed stacking the polymer backbones are randomly piled up. 
Table 1. Collected data from SEC analysis, absorption spectroscopy, cyclic voltammetry (CV) and photoelectron spectroscopy (PESA) measurements. Calculated HOMO and LUMO levels of the polymers.

\begin{tabular}{|c|c|c|c|c|c|c|c|c|c|c|}
\hline \multicolumn{2}{|l|}{ Polymer } & \multirow{2}{*}{$\begin{array}{l}\text { Molecular weight }^{a} \\
M n(\mathrm{da}) / \oplus\end{array}$} & \multicolumn{4}{|l|}{ Absorption data } & \multicolumn{2}{|c|}{ Redox potentials $(\mathrm{CV})^{\mathrm{e}}$} & \multicolumn{2}{|l|}{ Energy levels ${ }^{\mathrm{e}}$} \\
\hline & & & $\begin{array}{l}\lambda \max \text { solution }{ }^{b} \\
(\mathrm{~nm})\end{array}$ & $\begin{array}{l}\lambda \max \text { film } \\
(n m)\end{array}$ & $\begin{array}{l}\lambda \text { edge film } \\
(\mathrm{nm})\end{array}$ & $\begin{array}{l}\text { Eg (optical) } \\
(\mathrm{eV})\end{array}$ & $\begin{array}{l}\text { Eox } \\
(V)\end{array}$ & $\begin{array}{l}\text { Ered } \\
(V)\end{array}$ & $\begin{array}{l}\text { HOMO (CV) / } \\
\text { HOMO (PESA) } \\
(\mathrm{eV})\end{array}$ & $\begin{array}{l}\text { LUMO (CV) } \\
(\mathrm{eV})\end{array}$ \\
\hline \multirow[t]{2}{*}{ PF2 } & $\mathrm{Si}_{4}$ & $41000 / 2.4$ & $647^{c}$ & $449,635,698$ & 748 & 1.66 & 0.85 & - & $-5.25 /-5.07$ & \\
\hline & $\mathrm{C}_{20}$ & $38000 / 3.9$ & $546^{d}$ & $439,646,700$ & 756 & 1.64 & 0.95 & - & $-5.35 /-5.13$ & \\
\hline \multirow[t]{2}{*}{ PDPP-TT } & $\mathrm{Si}_{4}$ & $61000 / 3.0$ & 831 & 470,811 & 1008 & 1.23 & 0.60 & - & $-5.00 /-5.07$ & \\
\hline & $\mathrm{C}_{20}$ & $45000 / 6.0$ & 818 & 481,819 & 1019 & 1.21 & 0.80 & - & $-5.20 /-5.12$ & \\
\hline \multirow[t]{2}{*}{ PNDI-T $_{2}$} & $\mathrm{Si}_{4}$ & $61000 / 3.0$ & $623^{c}$ & $398,712,776$ & 865 & 1.43 & - & -0.71 & $-\quad /-5.70$ & -3.71 \\
\hline & $\mathrm{C}_{20}$ & $55000 / 3.9$ & $605^{d}$ & 395,702 & 832 & 1.49 & - & -0.58 & $-\quad /-5.78$ & -3.82 \\
\hline \multirow[t]{2}{*}{ РBTTT } & $\mathrm{Si}_{3}$ & $37000 / 1.6$ & 478 & 537 & 640 & 1.94 & 0.62 & - & $-5.02 /-4.94$ & \\
\hline & $C_{14}$ & $17000 / 2.9$ & 484 & 551 & 652 & 1.90 & 0.70 & - & $-5.10 /-4.81$ & \\
\hline
\end{tabular}

${ }^{a}$ Average molecular weight per number $(\mathrm{Mn})$ and dispersity $(\nexists)$ measured by high-temperature size exclusion chromatography $(\mathrm{SEC})$ at $150^{\circ} \mathrm{C}$ using 1,2,4-trichlorobenzene as an eluent and calibrated with narrow polystyrene standards; ${ }^{b}$ in $o$ DCB at room temperature otherwise mentioned;

${ }^{c}$ still aggregated at $95^{\circ} \mathrm{C}$ in $o \mathrm{DCB} ;{ }^{d}$ soluble at $95^{\circ} \mathrm{C}$ in $o$-DCB ; ${ }^{e}$ thin film measurements by cyclic voltammetry $(\mathrm{CV})$ or by photoelectron spectroscopy (PESA). 


\subsection{Thermal and structural properties}

The thermal stability of the polymers in nitrogen atmosphere was examined by thermogravimetric analysis (TGA) (figure S7a) and revealed similar stability data for all backbones and almost no influence of the side-chain nature. These materials are stable up to $230-280^{\circ} \mathrm{C}$; weight loss from degradation is detected at higher temperature and rapid decomposition occurs above $350^{\circ} \mathrm{C}$. The investigation of the phase behavior by differential scanning calorimetry (DSC) and polarizing optical microscopy (POM) confirmed the similarity of the polymers with siloxane and branched alkyl side-chains (figure S7b). All polymers display a frozen mesomorphic solid state that gradually flows to a fluid and birefringent mesophase on heating (figures S8). The mesophase extends to temperatures above $300^{\circ} \mathrm{C}$ until the transition to isotropic liquid state ( $T_{\text {iso }}$ ) for two of the branched alkyl side-chain polymers, while the third one (PDPP-TT-C20) and the three siloxane-based polymer analogues decompose before passing a transition (Table S7). Both PBTTT polymers also exhibit a fluid mesophase, with somewhat reduced $\mathrm{T}_{\text {iso }}$ close to $250^{\circ} \mathrm{C}$. However, the siloxane and linear alkyl side-chain polymers have different phase behaviors at low-temperature: PBTTT-Si3 freezes the mesophase in solid state, while PBTTT-C $\mathbf{1}_{\mathbf{1 4}}$ is in a crystallized state at room temperature and melts to mesophase around $40^{\circ} \mathrm{C}$.

The polymer self-assemblies were further investigated in bulk state at room and higher temperature by small- and wide-angle X-ray scattering (SWAXS) (figure S9). In fluid mesophase and in mesomorphic solid state, patterns contain a broad scattering signal from lateral distances between siloxane chains ( $h_{\mathrm{si}}$, maximum around $7 \AA$ ) or molten alkyl chains ( $h_{\text {ch }}$, maximum around 4.5-4.7 $\AA$ ) together with half-broad signal from $\pi$-stacking of backbones ( $h_{\pi}$, maximum between 3.5 and $4.1 \AA$ ) and with a series of sharp reflections ( $h 00$ ) of a lamellar periodicity. These signals are indicative of a smectic-like lamellar mesophase Lam(M), characterized by the alternation of stacked backbones and molten chain layers. $\mathbf{P F}_{2}$ and PNDI$\mathbf{T}_{2}$ polymers further exhibit sharp $(00 l)$ reflections implying the superimposition of same polymer backbone sub-units. This feature obviously results from the contribution of C-F bonds to lateral interactions between backbones in $\mathbf{P F}_{2}[36]$ and from the strong $\pi-\pi$ interaction between stacked NDI sub-units in PNDI-T2.[67] This observation is in perfect agreement with the segregated stacking mode (form 1) as illustrated in figure 5, previously deduced from UVvis results analysis. On the contrary, PDPP-TT patterns present no sign of sub-unit segregation, in line with previous results on PDPP-TT-C20[35] and observations made in UV-vis 
measurements pointing at a preferential mixed stacking (figure 5).[68] In addition to signals of lamellar and in-plane structures, one notices the appearance of secondary maximums on the wide-angle wing of $h_{\pi}$ for $\mathbf{P F}_{2}-\mathbf{S i}_{4}$ and PDPP-TT-Si4, which was attributed to $(h 2 l)$ reflections of an emerging three-dimensional structure. Regarding PBTTT-C14, the pattern at high temperature has the features of the $\operatorname{Lam}(\mathrm{M})$ phase, but at room temperature, $h_{\mathrm{ch}}$ signal gets a narrow shape and shifts to $4.4 \AA$, revealing the stiffening of molten chains in a compact crystalline conformation.

To elucidate the self-assembly, one needs to combine information from the patterns and the geometric characteristics of the repeat unit segments (table S9 and figure 6). Branched alkyl and siloxane chains have equivalent cross-sections $\sigma_{\mathrm{ch}}$ (see figure 1) which represent only 60 to $80 \%$ of the space requirement of the backbone repeat unit, as given by the product of repeat unit length $L_{\mathrm{ru}}$ and stacking distance $h_{\pi}$. For all polymers except PBTTT-C 14 , the conjugated repeat unit determines the degree of lateral expansion of lamellae with an average backbone spacing $h_{\mathrm{ru}}$ equal to $h_{\pi}$ (or slightly larger due to fluctuations), while molten chains adopt folded conformations to adjust their degree of lateral expansion to repeat unit area $A_{\text {ru. }}$ In the specific case of PBTTT-C 14 (the only polymer substituted with linear alkyl chain), $\sigma_{\mathrm{ch}}$ is much reduced and only represents $45 \%$ of $L_{\mathrm{ru}} \times h_{\pi}$ product. This discrepancy is hence, primarily compensated by the interdigitation of chains and secondarily by a conformation change. Moreover, the linear chains crystallize at room temperature into compact layers which in turn constrains the selfassembly of backbone rings, resulting in tilted structures with modified $\pi$-stacking distance and lamellar spacing.[69] Conversely, alkyl to siloxane chain substitution has a large impact on self-assembly of PBTTT, with in particular 16-19\% lateral expansion of lamellae and 5\% increase of $h_{\pi}$.
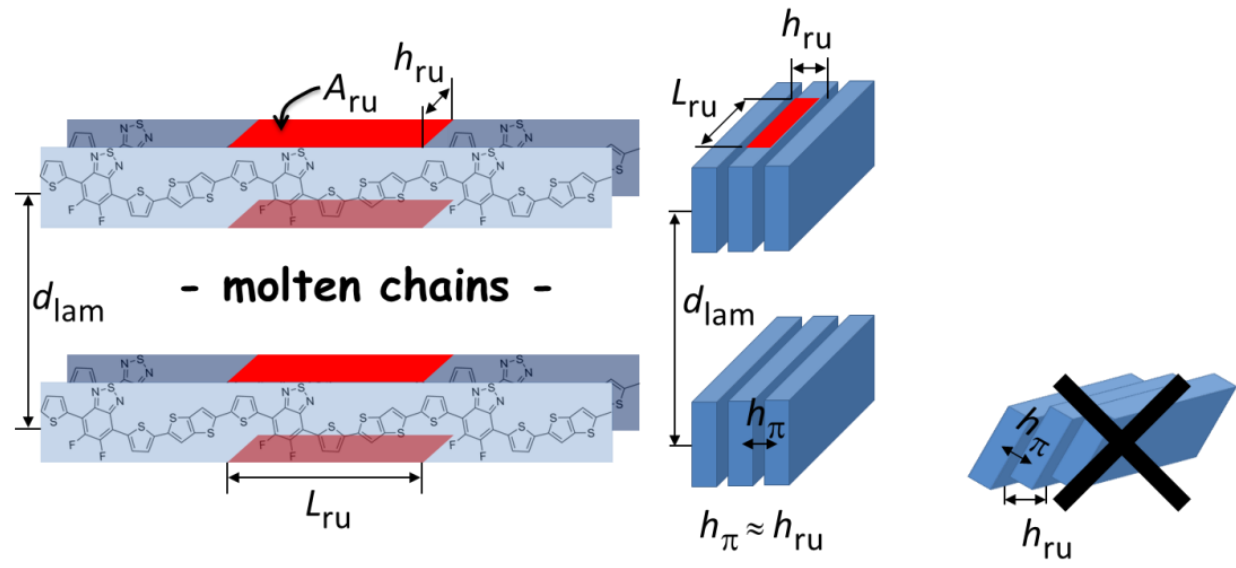
Figure 6. (Left) Schematic representation of lamellar system formed by conjugated polymers and definition of geometrical parameters $d_{\mathrm{lam}}, L_{\mathrm{ru}}, A_{\mathrm{ru}}$ and $h_{\mathrm{ru}}$. (Middle and right) View of the polymer lamellae along backbone, geometrical parameters and stacking distance $h_{\pi}$. Backbone planes are essentially vertical, leading to close $h_{\mathrm{ru}}$ and $h_{\pi}$ values (see text and table 2).

The structure and morphology of the polymers deposited as thin films in conditions of device fabrication were examined by GIWAXS (figure 7). All patterns display same structures as bulk polymers with equivalent self-assembly parameters (table 2). These structures are well aligned, with backbones and $c$-axis oriented parallel to surface. Scattering $h_{\mathrm{si}} / h_{\mathrm{ch}}$ of molten chains appear as a continuous broad ring, while the stiff and interdigitated chains of PBTTT-C14 give a narrow $h_{\mathrm{ch}}$ arc centered in the lamellar plane. Intensity of $h_{\pi}$ and reflections ( $\left.h 00\right)$ distribute within arcs or rings, with two preferential orientations: $h_{\pi}$ direction rather parallel to film for edge-on oriented domains and $h_{\square}$ direction rather normal to film for face-on oriented ones. The alkyl side-chain polymer films have comparable morphologies as in previous studies, consisting in equilibrated face-on/edge-on proportion for $\mathbf{P F}-\mathbf{C}_{20}$,[36] predominance of edge-on for PDPP-TT-C20,[65] essentially face-on for PNDI-T2-C20,[70] and essentially edge-on for PBTTT-C14.[71] The analogue siloxane side-chain polymers give same overall film morphologies, with the noticeable exception of PBTTT-Si3 giving a mixed face-on/edge-on orientation. Again, PBTTT-C14 stands out from the polymer series due to the compact layers of stiff, interdigitated chains that promote another alignment as the disentangled molten chain layers of PBTTT-Si3.

Due to their strong propensity to nanosegregate from other molecular segments (see section 2.1), siloxane side-chains form well-defined intermediate layers that improve the cohesion of the whole lamellar sequence, as otherwise confirmed by the extension of the Lam(M) range to higher (unreachable) temperatures (see figure S7b and table S7). This peculiarity is naturally expected to affect $\pi$-stacking interaction of backbones. One parameter of interaction strength consists in the average distance of $\pi$-stacked rings deduced from $h_{\pi}$ peak position. Actually, the impact of siloxane on $h_{\pi}$ turned out to be rather small: variation at the limit of significance for $\mathbf{P F}_{2}$ and slight decrease of 2-4\% (i.e. 0.1-0.15 ̊) for PDPP-TT and PNDI-T 2 (see tables 2 and figure S9). The larger $h_{\pi}$ value in the alkyl polymers PDPP-TT-C $\mathbf{C}_{20}$ and PNDI-T2-C20 is due to the proximal cumbersome branching point of the alkyl side-chain indeed, as evidenced by Lei et al.[72] and Han et al.[17] However, this effect is not observed in PF2-C20, that is attributed to the strong intermolecular coulombic dipole-dipole interactions provided by 
fluorine atoms maintaining a close $\pi$-stacking distance between polymer backbones whatever the side-chain nature is.[27,36,73] Another aspect of interaction strength is the degree of overlapping of facing backbones, which is in first approximation reproduced by the intensity of $h_{\pi}$ peak.[71] Intensity of (100) peak is a relevant reference since electronic densities of siloxane and alkyl chains coincide within 1-2\% and only the overall chain layer thickness needs to be considered: if $\pi$-stacking was not affected by siloxane chains, their introduction would reduce $I\left(h_{\pi}\right) / I(100)$ ratios by roughly $20 \%$. The experimental ratios as well as the accurate faceon/edge-on proportions were obtained from renormalized ring profiles (figure S10c). It turned out that ratios are not reduced, but increased by $100 \%$ for $\mathbf{P F}_{2}, 80 \%$ for PDPP-TT and $60 \%$ for PNDI-T2, revealing a substantial amplification of the $\pi$-stacking scattering. On the one hand, the greatly enhanced $\pi$-overlap of the polymer backbones induced by siloxane functionalization constitutes here an outcome of primary importance. On the other hand, film morphologies are also impacted since siloxane side-chain polymers gave systematically lower face-on proportions than branched alkyl analogues, although changes were only of 2 to $9 \%$. This observation likely arises from a side effect of the improved backbone overlap: intuitively, flatter layers should prefer contact to substrates favoring in-plane lamellar orientation. It has to be noted that opposite tendency (i.e. promotion of side-on lamellar orientation) was observed when laterally-attached trisiloxane side-chains were used.[16,17,21,23,24] In these latter cases however, the large volume variation caused by the branched siloxane segment should induce different polymer packing and lamellae configuration, ultimately leading to opposite polymer orientation trend.

Correlation length, as measured in the stacking direction $\left(\xi_{\text {para }}\right)$ and in the lamellae direction ( $\left.\xi_{\text {perp }}\right)$ constitute another point to discuss, as variable tendencies have already been published as a function of the alkyl-to-siloxane substitution.[16,24,28,31,74] For semiconducting polymers, indeed, $\xi_{\text {para }}$ is the most pertinent parameter, as it corresponds to the crystallite sizes as measured in the $\pi$-stacking planes. $\xi_{\text {perp }}$ is also a meaningful value in lamellar mesomorphic polymers, as it reflects the regularity of the lamellar layers on top of each other. Indeed, $\xi_{\text {para }}$ and $\xi_{\text {perp }}$ are accessible from $(0 k 0)$ and (100) peaks, respectively, and by using Scherrer equation. In this work, the variation of $\xi_{\text {para }}$ and $\xi_{\text {perp }}$ as a function of the alkyl-to-siloxane substitution gives variable results for the three polymer structures PF2, PDPP-TT and PNDI-T2. Interestingly, both values evolve in the same direction for PBTTT. Thus, $\xi_{\text {para }}$ and $\xi_{\text {perp }}$ are found to decrease in PF2-Si4, be comparable for PNDI-T2-Si4 or else, increase in PDPP-TT-Si4. In case of 
PBTTT polymer series, both correlation lengths are larger in PBTTT-C14, in agreement with its enhanced cohesive structure due to its crystallinity and interdigitated side-chains. It is interesting to notice that the $\pi$-stacking overlap enhancement, as observed in our siloxane systems, does not lead to a uniform increase of $\xi$ values, but depends on the very nature of polymer backbone. A possible explanation for the specific decrease of $\xi$ values in PF2-Si4 likely arises from the competing effect provided by the fluorine atoms and the siloxane sublayers which seek to impose their own polymer backbone stacking configuration, from dipolar interactions and segregation effects, respectively. In any case, it is worth mentioning that the correlation length values in mesomorphic polymers are naturally well below the range of interactions that typically lay in the micrometer scale, as evidenced by the polymer birefringence (figures $\mathrm{S} 8$ ).

In summary, siloxane side-chain polymers retain the overall structure and morphology of the branched alkyl side-chain analogues with only subtle variations of self-assembly parameters and domain orientations, whatever the nature of the bottom layer film. Although subtle but of utmost importance, one of these variations is the strong increase of the $\pi$-stacking overlap between conjugated backbones. As illustrated in figure 8, this behavior obviously arises from the highly fluid and nanosegregated siloxane sublayers that drive the lateral packing of the polymer backbones. Such polymer packing optimization is naturally expected to foster the efficiency of conduction pathways. Regarding the PBTTT polymer series, structural features are different and both materials are not comparable. Nevertheless, one notices that the crystallized and interdigitated alkyl chains of PBTTT-C $\mathbf{~}_{\mathbf{1 4}}$ lead to neat edge-on orientation and the molten siloxane chains of PBTTT-Si3 to mixed face-on/edge-on orientation. Such modified alignment behavior is not a surprise considering the change of structure type (semi-crystalline vs. mesomorphous) and of layer configuration, but its explanation would require other polymer couples investigation. At this stage, it is demonstrated that ramified alkyl chains can be replaced by siloxane chains with low impact on structure and morphology, which is not true for linear alkyl chains. 


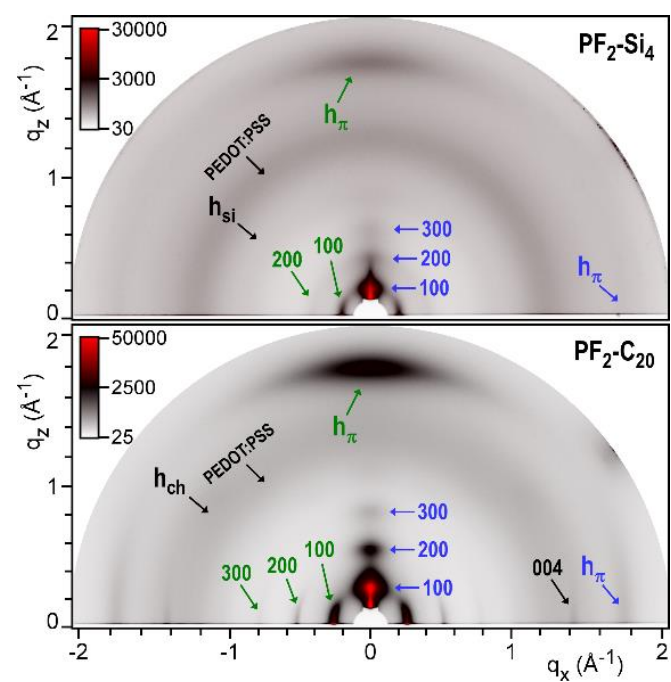

$\mathrm{a}$

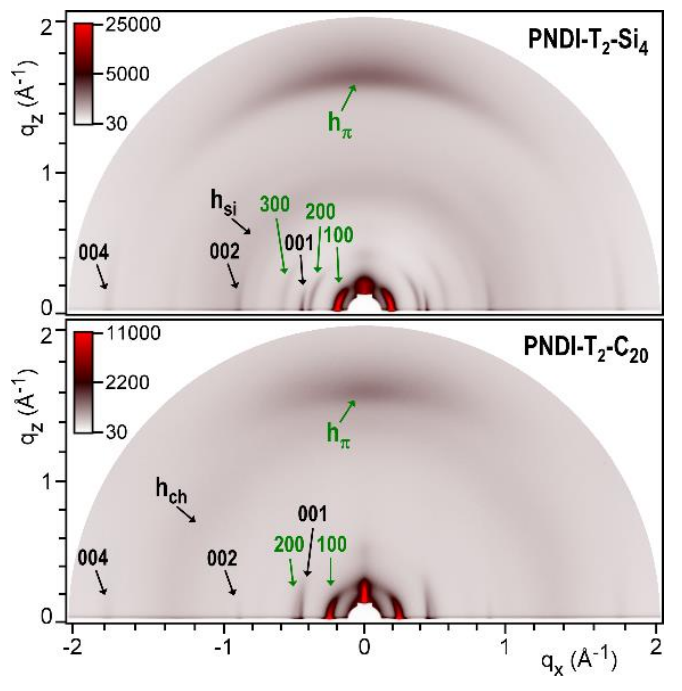

C

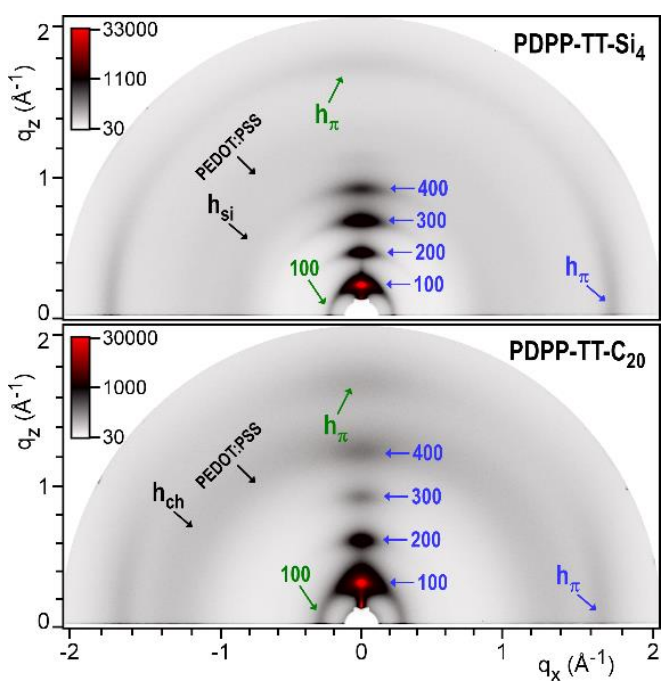

b

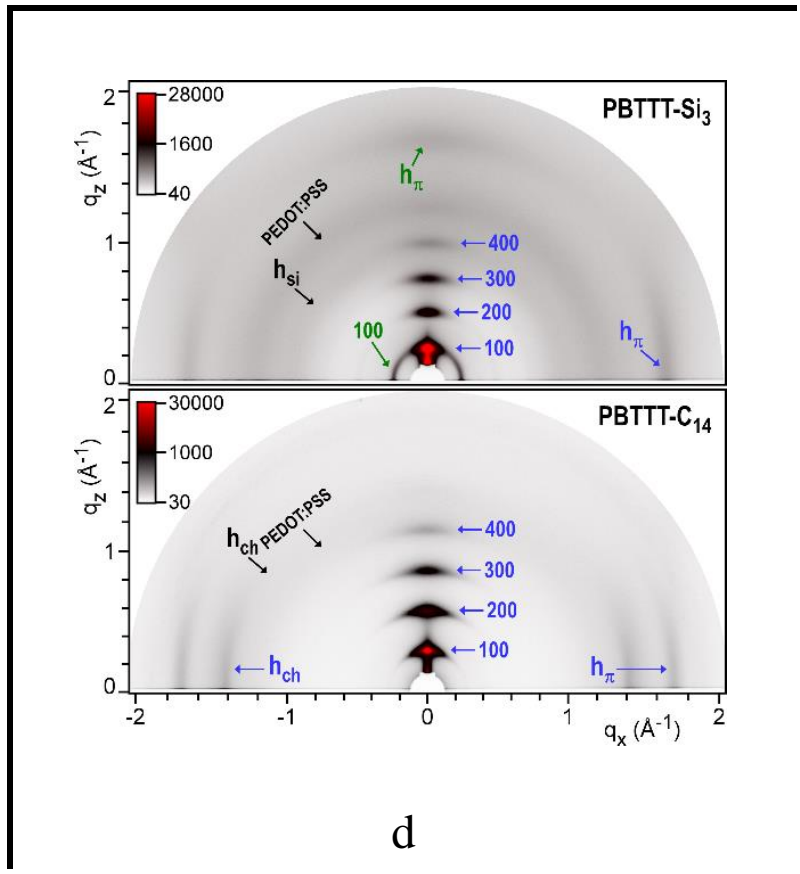

Figure 7. GIWAXS patterns of thin films of the 4 polymers with siloxane side-chains (top) and their alkyl side-chains counterparts (bottom), deposited on top of Si wafer with PEDOT:PSS bottom layer (PF 2 , PDPP-TT and PBTTT) or ZnO bottom layer (PNDI-T2). Polymers orient with backbone parallel to substrate (black labels) and various proportions of lamellae oriented rather parallel (blue labels for edge-on) and rather orthogonal to substrate (green labels for faceon). Reflections spots/arcs are pointed by Miller index labels $(h k l)$; scattering rings/arcs from stacking of backbones and from packing of siloxane and alkyl chains are respectively pointed by $h_{\pi}, h_{\mathrm{si}}$ and $h_{\mathrm{ch}}$ labels; scattering ring from PEDOT:PSS bottom layer is also indicated. GIWAX patterns with other bottom layers, as well as the patterns of the bare and bottom layer covered substrates are shown in ESI. 
Table 2. Structure and morphology of polymer thin films studied by GIWAXS, as obtained from patterns and analyses presented in figures 7 and S10a,c.

\begin{tabular}{|c|c|c|c|c|c|c|c|}
\hline \multicolumn{2}{|l|}{ Polymer } & Phase ${ }^{a}$ & $d_{\mathrm{lam}}\left(\xi_{\text {perp }}\right)^{\mathrm{b}}$ & $q_{\mathrm{ch}}\left(n_{\mathrm{chL}}\right)^{\mathrm{c}}$ & $h_{\pi}\left(\xi_{\text {para }}\right)^{d}$ & $I\left(h_{\pi}\right) / /(100)^{\mathrm{e}}$ & face-on $(\%)^{f}$ \\
\hline \multirow[t]{2}{*}{$\mathrm{PF}_{2}$} & $\mathrm{Si}_{4}$ & Lam(M) & $30.2(120)$ & $1.52(2)$ & $3.54(35)$ & $55 \mathrm{~g}$ & $49 \mathrm{~g}$ \\
\hline & $\mathrm{C}_{20}$ & Lam(M) & $23.7(190)$ & $1.48(2)$ & $3.51(45)$ & $27 \mathrm{~g}$ & $58 \mathrm{~g}$ \\
\hline \multirow[t]{2}{*}{ PDPP-TT } & $\mathrm{Si}_{4}$ & Lam(M) & $27.2(150)$ & $1.67(2)$ & $3.60(50)$ & $53 / 55 / 48^{h}$ & $14 / 14 / 11^{h}$ \\
\hline & $\mathrm{C}_{20}$ & Lam(M) & $20.3(105)$ & $1.71(2)$ & $3.75(35)$ & $29 / 32 / 28 \mathrm{~h}$ & $16 / 16 / 18^{h}$ \\
\hline \multirow[t]{2}{*}{ PNDI-T 2} & $\mathrm{Si}_{4}$ & Lam(M) & $32.1(215)$ & $1.43(2)$ & $3.80(35)$ & $23 / 21^{i}$ & $87 / 91^{i}$ \\
\hline & $\mathrm{C}_{20}$ & Lam(M) & $25.4(190)$ & $1.38(2)$ & $3.90(35)$ & $14 / 14^{i}$ & $95 / 96^{i}$ \\
\hline \multirow[t]{2}{*}{ PBTTT } & $\mathrm{Si}_{3}$ & Lam(M) & $25.2(120)$ & $1.29(2)$ & $3.78(45)$ & $32 \mathrm{~g}$ & 189 \\
\hline & $C_{14}$ & $\operatorname{Lam}(\mathrm{Cr})$ & $21.9(185)$ & $1.14(1)$ & $3.67(70)$ & 449 & $1.6 \mathrm{~g}$ \\
\hline
\end{tabular}

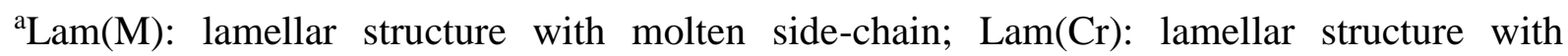
crystallized side-chains. ${ }^{\mathrm{b}} d_{\mathrm{lam}}$ : lamellar periodicity from $(h 00)$ reflection series, and $\xi_{\text {perp: }}$ : correlation length from (100) reflection width and Scherrer equation with shape factor $K=0.9$ $(\AA) .{ }^{c} q_{\mathrm{ch}}=\left(n_{\mathrm{chL}} / 2\right) \times A_{\mathrm{ru}} / \sigma_{\mathrm{ch}}$ : chain packing ratio (i.e. ratio of layer area and cross-sectional area per chain), with $n_{\mathrm{chL}}=1$ for monolayers of interdigitated chains and $n_{\mathrm{chL}}=2$ for bilayers of disentangled chains. ${ }^{\mathrm{d}} h_{\pi}: \pi$-stacking distance of backbones $(\AA)$, and $\xi_{\text {para: }}$ correlation length of stacking from Scherrer equation with $K=0.9(\AA)$. ${ }^{\mathrm{e}} \% I\left(h_{\pi}\right) / I(100)$ intensity ratio of $h_{\pi}$ and reflection (100) (figure S10c). ${ }^{\mathrm{f}} \%$ face-on is the proportion of face-on domains, defined as those whose $\pi$-stacking direction is tilted by less than $45^{\circ}$ from film normal (figure $\mathrm{S} 10 \mathrm{c}$ ); ${ }^{\mathrm{g}}$ deposited on PEDOT:PSS bottom layer; hdeposited either on PEDOT:PSS bottom layer, on bare substrate with native $\mathrm{SiO}_{2}$ layer or on HMDS bottom layer; ${ }^{\mathrm{i}}$ deposited either on $\mathrm{ZnO}$ or on HMDS bottom layer.

a)

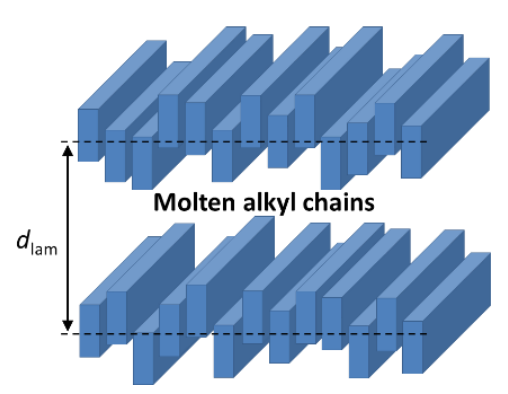

b)

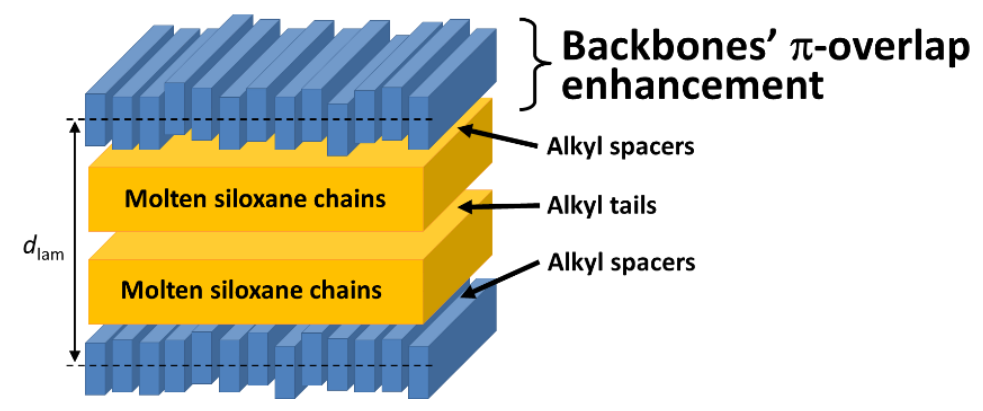

Figure 8 . Illustration of the role of the linear siloxane hybrid side-chains in the $\pi$-stacking overlap enhancement of the conjugated polymer backbones (here drawn to scale for PF2 
polymer series). The strong segregation propensity of siloxanes associated to their remarkable fluidity improve the lateral packing efficiency of the conjugated backbones.

\subsection{Charge transport properties}

In organic semiconducting polymers, charge transport properties are not only strongly related to the frontier molecular orbitals (HOMO and LUMO, respectively), but also to the solid-state structural properties and the polymer backbone orientation as regards to the substrates. In this respect, given the significant structural variations noted in the previous analyses, alkyl and siloxanes polymers are expected to exhibit different charge transport properties.

Charge carrier mobilities have been determined in the different polymers using two types of devices. The mobility in the substrate plane was estimated from the transfer characteristics of bottom-gate/bottom contact (BG/BC) organic field-effect transistors (OFETs). The chargecarrier mobility in the direction perpendicular to the substrate was extracted from the (I-V) characteristics of hole-only or electron-only diodes showing space-charge limited current (SCLC). The detailed elaboration and characterization procedure for each type of device can be found in the supporting information. While a strong evolution of the charge-carrier mobility was observed between as-cast devices and devices annealed at $120^{\circ} \mathrm{C}$ for 15 minutes, almost no evolution was observed after a subsequent isochronal annealing at $150^{\circ} \mathrm{C}$. Consequently, hole and electron mobilities measured after a thermal annealing at $120^{\circ} \mathrm{C}$ for 15 minutes (see table 3 and figure 9) will be discussed in the following.

Table 3. OFET and SCLC charge carrier mobility.

\begin{tabular}{|l|l|c|c|}
\hline \multicolumn{2}{|l|}{ Polymers } & $\mu_{\text {OFET }}\left(\mathrm{cm}^{2} / \mathrm{V} . \mathrm{s}\right) \times 10^{-3}$ & $\mu_{\text {SCLC }}\left(\mathrm{cm}^{2} / \mathrm{V} . \mathrm{s}\right) \times 10^{-3}$ \\
\hline \multirow{3}{*}{ PF2 } & $\mathbf{S i}_{4}$ & $90 \pm 10$ & $6.9 \pm 0.2$ \\
\cline { 2 - 4 } & $\mathbf{C}_{20}$ & $30 \pm 3$ & $4.1 \pm 0.5$ \\
\hline \multirow{2}{*}{ PDPP-TT } & $\mathbf{S i}_{4}$ & $500 \pm 100$ & $12 \pm 1$ \\
\cline { 2 - 4 } & $\mathbf{C}_{20}$ & $230 \pm 30$ & $5.9 \pm 0.6$ \\
\hline \multirow{2}{*}{ PBTTT } & $\mathbf{S i}_{4}$ & $23 \pm 3^{*}$ & $1.2 \pm 0.1^{*}$ \\
\cline { 2 - 4 } & $\mathbf{C}_{20}$ & $13 \pm 1^{*}$ & $0.19 \pm 0.01^{*}$ \\
\cline { 2 - 4 } & $\mathbf{S i}_{3}$ & $2.4 \pm 0.3$ & $6.4 \pm 0.9$ \\
\cline { 2 - 4 } & $\mathbf{C}_{14}$ & $160 \pm 20$ & $0.47 \pm 0.08$ \\
\hline
\end{tabular}

* corresponds to electron mobility 
As evidenced in table 3 and figure 9, the substitution of branched alkyl chains $\left(\mathbf{C}_{\mathbf{2 0}}\right)$ by linear siloxane chains ( $\mathbf{S i} \mathbf{i})$ leads to a systematic increase of OFET and SCLC mobility. Thus, the hole OFET mobility is multiplied by 2 to 3 when going from PF2-C20 to PF2-Si4 or from PDPPTT-C20 to PDPP-TT-Si4. The same holds for the electron OFET mobility which is multiplied by almost 2 when going from PNDI-T2-C20 to PNDI-T2-Si4. The very same trend is observed for the SCLC mobility. In the saturation regime, OFET hole mobility as high as $10^{-1} \mathrm{~cm}^{2} / \mathrm{V} . \mathrm{s}$ and $5 \times 10^{-1} \mathrm{~cm}^{2} /$ V.s were measured on PF2-Si4 and PDPP-TT-Si4 OFETs, respectively. Such values together with an electron mobility of $2.3 \times 10^{-2} \mathrm{~cm}^{2} / \mathrm{V}$.s for PNDI-T2-Si4 are remarkably high for simple (BG/BC) OFETs.

The increase in charge-carrier mobility is fully consistent with GIWAXS measurements. Indeed, on the one hand, the substitution of branched alkyl chain by linear siloxane chains leads to a significant increase in the $\pi$-overlap of the conjugated polymer backbone stacks (figure 8). On the other hand, the overall film morphology (edge-on/face-on ratio) is rather unaffected by this side-chain modification. The improved charge transport measured in both device configurations and for the three backbone types is therefore explained by a better efficiency of conduction pathways that follows the increased $\pi$-overlap. In the specific case of PNDI polymer series, one notices a relatively low SCLC mobility (especially for PNDI-T2-C20) despite its high ratio of side-on orientation. This result can also be explained by the low $\pi$-stacking overlap (see Table 2). A possible reason for such low overlap could be that although the polymer stacking is segregated (see figure 5), it should be notably shifted along the backbone to adjust the packing of the two comonomers of quite different size. The PBTTT polymer series has to be commented separately as different side-chains are inserted, i.e. a linear alkyl chain (PBTTT$\mathbf{C}_{14}$ ) and a short linear siloxane chain (PBTTT-Si3). Inevitably, the large molecular section discrepancy of these chains (see figure 1) results in a significant change in lamellar configuration and solid-state morphology. As seen in previous section 2.4, PBTTT-C14 stands out from the polymer series with its crystalline state (with interdigitated chains), a better backbone $\pi$-overlap and a neat edge-on polymer orientation, while PBTTT-Si3 is mesomorphic and adopts a mixed edge-on/face-on orientation. For charge carrier properties, we thus observe a much smaller OFET hole mobility for PBTTT-Si3 than for PBTTT-C 14 (divided by more than 60), following the concomitant loss of crystallinity, reduction of $\pi$-stacking overlap and edge-on destabilization in PBTTT-Si3. 
As SCLC diodes probe the charge-carrier transport in the direction perpendicular to the substrate, the hole SCLC mobility in PBTTT-C $\mathbf{1}_{\mathbf{1 4}}$ is particularly low as this polymer is purely edge-on in the solid-state. The very large OFET to SCLC mobility ratio (over 300) observed for PBTTT-C 14 contrasts with the rather equilibrated in-plane and out-of-plane mobility of PBTTT-Si3. This latter polymer, that exhibits a moderate but higher perpendicular hole mobility, might then constitute a good candidate to be used as a high bandgap electron donor component for organic photovoltaics.

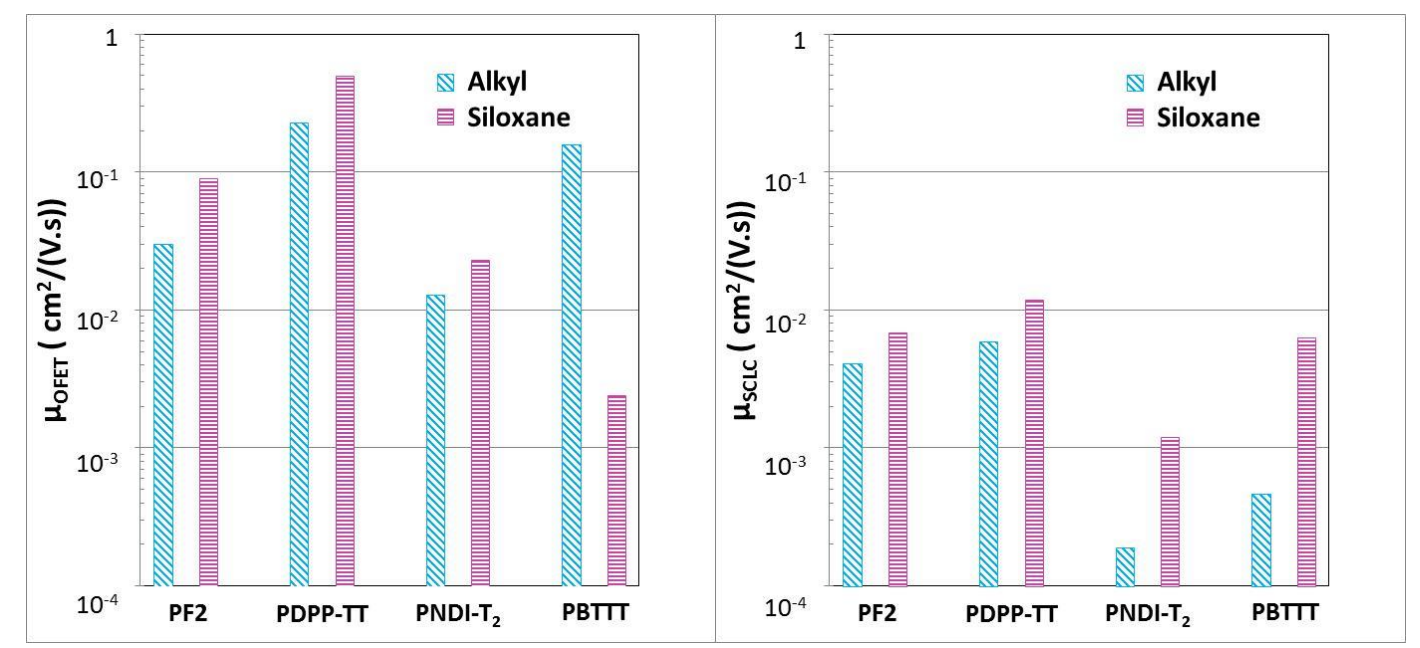

Figure 9. Comparison of charge carrier mobility of the polymers, as measured on OFETs (left) or SCLC diodes (right) after annealing at $120^{\circ} \mathrm{C}$ for 15 minutes.

\section{Conclusion}

Four different $\pi$-conjugated polymer structures have been functionalized by linear siloxane hybrid side-chains. The latter was a tetrasiloxane $\left(\mathbf{S i}_{4}\right)$ or a trisiloxane $\left(\mathbf{S i}_{3}\right)$ linear segment connected via its extremity to a pentylene linker. The polymer structures were selected for their excellent charge carrier transport properties, i.e. based on a 5,6-difluorobenzothiadiazole unit (PF2), a diketopyrrolopyrrole unit (PDPP-TT), a naphtalediimide unit (PNDI-T2) and a poly[bis(thiophen-2-yl)thieno[3,2,b]thiophene (PBTTT). The properties of these siloxanefunctionalized polymers were scrutinized and compared with the ones of their alkyl-substituted polymer analogues.

The impact of the alkyl-to-siloxane chain substitution clearly depends on the molecular section of the side-chains. When ramified alkyl chains (2-octyldodecyl: $\mathbf{C}_{20}$ ) were replaced by linear siloxane chains of equivalent cross section $(\mathbf{S i} 4)$, the structure and film morphology were 
essentially preserved, which was not true when alkyl and siloxane chains were both linear and thus had different cross-sections ( $\mathbf{C}_{14}$ and $\mathbf{S i}$ ). In this latter case, layer configurations, domain orientations and even structure types (semi-crystalline vs. smectic-like) were changed, rendering any comparison senseless. The other siloxane side-chain polymers differed from analogue alkylated polymers (i.e. of similar molecular section) by a considerable enhancement of charge transport properties, in accordance with findings reported for previous polymers. In contradiction with speculative explanations given in literature, morphology obviously played no significant role since it was essentially maintained and since charge transport was improved for both, out-of-plane and in-plane conductions. Explanations based on a reduced $\pi$-stacking distance reinforcing the interactions are also unrealistic, since we found a small or even an insignificant distance change. On the contrary, we observed for the first time an effect on the scattering intensity from $\pi$-stacking, as the scattering was considerably amplified by 60 to $100 \%$. This reveals a substantially higher overlapping of conjugated backbones which clearly contributes to the $\pi$-stacking interactions strength and to the conduction pathways efficiency. Consequently, the improved charge transport mainly originates from this higher $\pi$-overlap, what we believe was also true but overlooked for previous siloxane side-chain polymers. Our conviction relies on the particular architecture of these polymers and on the intrinsic propensity of siloxane for segregation. Siloxane chains were indeed always connected to backbones by short alkyl spacers and the formation of the well-segregated, flat siloxane layers inevitably drove the lateral packing of the backbones toward a higher overlap.

To sum up, this study provides a complete description of the alkyl-to siloxane chain substitution effect scenario on the properties of semiconducting polymers. Provided that the molecular areas of the side-chains are comparable, the impact of siloxane chains becomes intimately linked to their unique chemical nature. In the main, oligodimethylsiloxanes exhibit a remarkable fluidity, very hardly crystallize even at very low temperature, and show a strong propensity for segregation. As a result, siloxane-functionalized conjugated polymers exhibit a well-defined lamellar mesomorphic organization that is stabilized over a very broad temperature range (both on the very low and very high temperature sides). The lamellar structure is endowed with a relative fluidity, and the lamellae formed by the polymer stacks are allowed to glide the one on the others. As revealed in the present study, the siloxane layers also drive the packing of the polymers by imposing a better facing of the polymer backbones: the $\pi$-stacking polymer overlap is significantly enhanced. This brings a clear demonstration of the impact of the side-chain nature on the degree of $\pi$-stacking of conjugated cores. Such effect is obviously of tremendous 
importance for charge carrier mobility, that is actually found to increase significantly (both in the plane and out of the plane) in siloxane-functionalized polymers. These results pave the way to the future development of linear siloxane substituted polymers and of new highly-efficient semiconducting materials by controlled-siloxane functionalization.

\section{Experimental Section}

Supporting information provide the details of the synthesis and the characterization data, including all plots obtained by size exclusion chromatography (SEC), temperature-dependence absorption spectroscopy, cyclic voltammetry (CV), thermogravimetry (TGA), differential scanning calorimetry (DSC), polarized optical microscopy (POM), photoelectron spectroscopy (PESA), X-ray scattering (SWAXS and GIWAXS), OFET and SCLC measurements, NMR and mass spectrometry.

\section{Conflict of Interest.}

The authors declare no conflict of interest

\section{Acknowledgments}

The authors gratefully acknowledge Dr. Fanny Richard and Pr. Paolo Samori (ISIS, Strasbourg) for carrying out PESA measurements, Dr. Jean-Marc Strub (IPHC, Strasbourg) for carrying out mass spectrometry measurements, and Emilie Voirin for her assistance in synthesis. They also gratefully acknowledge the different following funding programs. INTERREG Upper Rhine (European Regional Development Fund), the Ministries for Research of Baden-Württemberg, Rheinland-Pfalz and the Region Grand Est provided a funding in the framework of the Science Offensive Upper Rhine program (PROOF project). A support was received from the "PEPS cellule Energie 2019" program from CNRS. This work was also funded by the French National Research Agency (ANR) through the Programme d'Investissement d'Avenir under contract ANR-11-LABX-0058_NIE within the Investissement d'Avenir program ANR-10-IDEX-000202. The authors also thank Pohang Accelerator Laboratory (PAL) for giving the opportunity to perform the GIWAXS measurements, MEST and POSTECH for supporting these experiments, Dr Hyungju Ahn for adjustment and help, and other colleagues from the 9A USAXS beamline for assistance. 


\section{References}

[1] a) Z.-G. Zhang, Y. Li, Science China: Chem. 2015, 58, 192 ; b) L. Shi, Y. Guo, W. Hu, Y. Liu, Mater. Chem. Front. 2017, 1, 2423 ; c) T. Marszalek, M. Li, W. Pisula, W, Chem. Commun. 2016, 52, 10938 ; d) Z. Liu, G. Zhang, D. Zhang, Acc. Chem. Res. 2018, 51, 1422 ; e) T. Lei, J.-Y. Wang, J. Pei, Acc. Chem. Res. 2014, 47, 1117 ; f) Z. Yi, S. Wang, Y. Liu, Adv. Mater. 2015, 27, 3589 ; g) Z. He, D. Li, D. K. Hensley, A. J. Rondinone, J. Chen, Appl. Phys. Lett. 2013, 103, 113301.

[2] L. Biniek, S. Fall, C. L. Chochos, D. V. Anokhin, D. Ivanov, N. Leclerc, P. Lévêque, T. Heiser, Macromolecules, 2010, 43, 9779.

[3] L. Huang, G. Zhang, K. Zhang, Q. Peng, M. S. Wong, Macromolecules, 2019, 52, 4447.

[4] J. Cho, S. H. Yu, D. S. Chung, J. Mat. Chem. C 2017, 5, 2745.

[5] J. E. Houston, S. Richeter, S. Clément, R. C. Evans, Polym Int. 2017, 66, 1333.

[6] J. Freudenberg, D. Jänsch, F. Hinkel, U. H. F. Bunz, Chem. Rev. 2018, 118, 5598.

[7] Z. Liu, G. Zhang, D. Zhang, Acc. Chem. Res. 2018, 51, 1422.

[8] M. Goal, C. D. Heinrich, G. Krauss, M. Thelakkat, Macromol. Rapid Commun. 2019, 40, 1800915.

[9] T. L. Nguyen, C. Lee, H. Kim, Y. Kim, W. Lee, J. H. Oh, B. J. Kim, H. Y. Woo, Macromolecules, 2017, 50, 4415.

[10] C. B. Nielsen, A. Giovannitti, D.-T. Sbircea, E. Bandiello, M. R. Niazi, D. A. Hanifi, M. Sessolo, A. Amassian, G. G. Malliaras J. Rivnay, I. McCulloch, J. Am. Chem. Soc. 2016, 138, 10252.

[11] B. Meng, H. Song, X. Chen, Z. Xie, J. Liu, L. Wang, Macromolecules 2015, 48, 4357.

[12] X. Liu, C. Zhang, C. Duan, M. Li, Z. Hu, J. Wang, F. Liu, N. Li, C. J. Brabec, R. A. J. Janssen, G. C. Bazan, F. Huang, Y. Cao, J. Am. Chem. Soc. 2018, 140, 8934.

[13] a) K. Miao, G.J. Chae, X. Wu, Q. Shu, X. Zhu, B. Sun, J. Fan, S. Cho, RSC Adv. 2016, 6, 29164 ; b) B. Kang, R. Kim, S. B. Lee, S.-K. Kwon, Y.-H. Kim, K. Cho, J. Am. Chem. Soc. 2016, 138, 3679.

[14] H.-G. Jeong, B. Lim, D. Khim, M. Han, J. Lee, J. Kim, J.-M. Yun, K. Cho, J.-W. Park, D.-Y. Kim, Adv. Mater. 2013, 25, 6416. 
[15] P. Homyak, Y. Liu, S. Ferdous, F. Liu, T. P. Russeell, E. B. Coughlin, Chem. Mater. 2015, 27, 443.

[16] J. Mei, D. H. Kim, A. L. Ayzner, M. F. Toney, Z. Bao, J. Am. Chem. Soc. 2011, 133, 20130.

[17] A.-R. Han, J. Lee, H. R. Lee, J. Lee, S.-H. Kang, H. Ahn, T. J. Shin, J. H. Oh, C. Yang, Macromolecules, 2016, 49, 3739.

[18] J. Lee, A.-R. Han. J. Kim, Y. Kim, J. H. Oh, C. Yang, J. Am. Chem. Soc. 2012, 134, 20713.

[19] X. Liu, L. Nian, K. Gao, L. Zhang, L. Qing, Z. Wang, L. Ying, Z. Xie, Y. Ma, Y. Cao, F. Liu, J. Chen, J. Mat. Chem. A 2017, 5, 17619.

[20] S. Feng, C. Liu, X. Xu, X. Liu, X. Zhang, Y. Nian, Y. Cao, J. Chen, ACS Macro. Lett. 2017, 6, 1310 .

[21] G. H. L. Heintges, K. H. Hendriks, F. J. M. Colberts, M. Li, J. Li, R. A. J. Janssen, RSC Adv. 2019, 9, 8740 .

[22] I. Ohnishi, K. Hashimoto, K.Tajima, R. Soc. Open Sci. 2018, 5, 172025.

[23] D. H. Kim, A.L. Ayzner, A. L. Appleton, K. Schmidt, J. Mei, M. F. Toney, Z. Bao, Chem. Mater. 2013, 25, 431.

[24] C. Grand, S. Baek, T.-H. Lai, N. Deb, W. Zajaczkowski, R. Stalder, K. Müllen, W. Pisula, D. G. Bucknall, F. So, J. R. Reynolds, Macromolecules 2016, 49, 4008.

[25] Y. Kim, D. X. Long, J. Lee, G. Kim, T. J. Shin, K.-W. Nam, Y.-Y. Noh, C. Yang, Macromolecules 2015, 48, 5179.

[26] Y. An, D. X. Long, Y. Kim. Y.-Y. Noh, C. Yang, Phys. Chem. Chem. Phys. 2016, 18, 12486.

[27] Y. Li, S. Song, S. Y. Park, J. Y. Kim, H. Y. Woo, Science China: Chemistry. 2017, 60.

[28] H. Jiang, F. Pan, L. Zhang, X. Zhou, Z. Wang, Y. Nian, C. Liu, W. Tang, Q. Ma, Z. Ni, M. Chen, W. Ma, Y. Cao, J. Chen, ACS Appl. Mater. \& Interfaces 2019, 11, 29094.

[29] B. Fan, L. Ying, P.Zhu, F. Pan, F. Liu, J. Chen, F. Huang, Y. Cao, Adv. Mater. 2017, 1703906. 
[30] Q. Wang, Z. Hu, Y. Lin, L. Zhang, L. Liu, Y. Ma, Y. Cao, J. Chen, ACS Appl. Mater. \& Interfaces 2020, 12, 4659.

[31] Z. Tang, X. Xu, R. Li, L. Yu, L. Meng, Y. Wang, Y. Li, Q. Peng, ACS Appl. Mater. \& Interfaces 2020, 12, 17760.

[32] J. Mei, H.-C. Wu, Y. Diao, A. Appletonn, H. Wang, Y. Zhou, W.-Y. Lee, T. Kurosawa, W.-C. Chen, Z. Bao, Adv. Funct. Mater. 2015, 25, 3455.

[33] J. Lee, A.-R. Han, H. Yu, T. J. Shin, C. Yang, J. H. Oh, J. Am .Chem. Soc. 2013, 135, 9540.

[34] Y. Ding, F. Zhao, S. Kim, X. Wang, H. Lu, G. Zhang, K. Cho, L. Qiu, ACS Appl. Mater. \& Interfaces, DOI :10.1021/acsami.0c11436.

[35] Y. Li, S. P. Singh, P. A. Sonar, Adv. Mater. 2010, 22, 4862.

[36] O. A. Ibraikulov, C. Ngov, P. Chávez, I. Bulut, B. Heinrich, O. Boyron, K. L. Gerasimov, D. A. Ivanov, S. Swaraj, S. Méry, N. Leclerc, P. Lévêque, T. Heiser, J. Mat. Chem. A 2018, 6, 12038.

[37] M. Xiaoling, A. Qiaoshi, O. A. Ibraikulov, P. Lévêque, T. Heiser, N. Leclerc, X. Zhang F. Zhang, J. Mat. Chem. A 2020, 8, 1265.

[38] H. Yan, Z. Chen, Y. Zheng, C. Newman, J. R. Quinn, F. Dötz, M. Kastler, A. Facchetti, Nature, 2009, 457, 679.

[39] I. McCulloch, M. Heeney, C. Bailey, K. Genevicius, I. McDonald, M. Shkunov, D. Sparrowe, S. Tierney, R. Wagner, W. Zhang, M. L. Chabinyc, R. J. Kline, M. D. McGehee, M. F. Toney, Nature Mat. 2006, 5, 328.

[40] A. C. M. Kuo, Poly(dimethylsiloxane) in Polymer Data Handbook, Oxford Univ. Press. Ed. J. E. Mark, 1999.

[41] J.-E. Marks, Acc. Chem. Res. 2004, 37, 946.

[42] E. Yilgör, I. Yilgör, Prog. Polym. Sci. 2014, 39, 1165.

[43] M. J. Owen, Surface properties and applications, chapter 8 in Silicon-containing polymers. Eds. R.J. Jones and al. Kluwer Acad. Publishers. 2000.

[44] a) M. L. Hammock, O. Knopfmacher, T. Nga Ng, J. B.-H. Tok, Z. Bao, Adv. Mater. 2014, 26, 6138 ; b) O. Knopfmacher, M. L. Hammock, A. L. Appleton, G. Schwartz, J. Mei, T. Lei, J. Pei, Z. Bao, Nature Commun. 2014, 5, 2954. 
[44] K. Panneerselvam, M. P. Antony, T. G. Srinivasan, P. R. Vasudeva Rao, Thermochimica Acta, 2007, 466, 49.

[45] J. Ng Lee, C. Park, G. M. Whitesides, Anal. Chem. 2003, 75, 6544.

[46] Z. Küçükyavuz, N. Al-Ghezawi, Eur. Polym. J. 1990, 26, 653.

[47] P. R. Dvornic, Thermal properties of polysiloxanes, chapter 7 in Silicon-containing polymers. Eds. Jones R. J. and al. Kluwer Acad. Publishers. 2000.

[48] S. W. Sides, J. Curro, G. S. Grest, M. J. Stevens, T. Soddemann, A. Habenschuss, J. D. Londono, Macromolecules, 2002, 35, 6455.

[49] S. J. Clarson, K. Dodgson, J. A. Semlyen, Polymer, 1985, 39, 930.

[50] Polymer Handbook, $4^{\text {th }}$ ed., edited by Brandrup, J. Immergut, E. H. Grulke; E. A. Chap VI. John Wiley \& Sons, 1999.

[51] P. J. Flory, H. Shih, Macromolecules, 1972 5, 761.

[52] G. Belorgey, G. Sauvet, Organosiloxane block and graft copolymers, chapter 2 in Silicon-containing polymers. Eds. Jones R. J. and al. Kluwer Acad. Publishers. 2000.

[53] E. Pouget, J. Tonnar, P. Lucas, P. Lacroix-Demazes, F. Ganachaud, B. Boutevin, Chem. Rev. 2010, 110, 1233.

[54] A. Ciolino, G. Sakellariou, D. Pantazis, M. A. Villar, E. Valle, N. Hadjichristidis, J. Polym. Sci.: Part A Polym. Chem. 2006, 44, 1579.

[55] M. A. Semsarzadeh, M. Ghahramani, M. J. Memb. Sci. 2020, 594, 117400.

[56] D. Guillon, M. A. Osipov, S. Méry, M. Siffert, J.-F. Nicoud, C. Bourgogne, P. Sebastião, J. Mat. Chem. 2001, 11, 2700.

[57] R. Kieffer, M. Prehm, K. Pelz, U. Baumeister, F. Liu, H. Hahn, H. Lang, G. Ungar, C. Tschierske, Soft Matter 2009, 5, 1214.

[58] M. S. Motawia, J. Wengel, A. E. E. Abdel-Megid, E. B. Pedersen, Synthesis, 1989, 384.

[59] M. Waugaman, B. Sannigrahi, P. McGeady, I. M. Khan, Eur. Pol. J. 2003, 39, 1405.

[60] A. P. Kulkarni, C. J. Tonzola, A. Babel, S. A. Jenekhe, Chem. Mater., 2004, 16, 4556.

[61] a) C. Zhang, Z. Chen, C. Yang, E. Liang, J. Yi, G. Yu, C. Yang, Macromol. Chem. Phys. 2018, 219, 1700474 ; b) S.-F. Yang, Z.-T. Liu, Z.-X. Cai, H.-W. Luo, P.-L. Qi, G.-X. Zhang, D.-Q. Zhang, Macromolecules 2016, 49, 5857. 
[62] L. Biniek, S. Fall, C. L. Chochos, N. Leclerc, P. Lévêque, T. Heiser, Org. Elect. 2012, $13,114$.

[63] Y. Zhong, L. Biniek, N. Leclerc, S. Ferry, M. Brinkmann, Macromolecules 2018, 51, 4238.

[64] M. Brinkmann, E. Gonthier, S. Bogen, E. K. Tremel, S. Ludwigs, M. Hufnagel, M. Sommer, ACS Nano 2012, 6, 10319.

[65] L. Biniek, N. Leclerc, R. Bechara, T. Heiser, M. Brinkmann, Macromolecules, 2013, $46,4014$.

[66] K. Tremel, F. S. U. Fischer, N. Kayunkid, R. Di Pietro, R. Tkachov, A. Kiriy, D. Neher, S. Ludwigs, M. Brinkmann, Adv. Energy Mater., 2014, 4, 1301659.

[67] Z. Chen, M. Lee, J. R. S. Ashraf, Y. Gu, S. Albert-Seifried, M. M. Nielsen, B. Schroeder, T. D. Anthopoulos, M. Heeney, I. McCulloch, H. Sirringhaus, Adv. Mater. 2012, 24, 647 .

[68] L.-H. Li, O. Y. Kontsevoi, S. H. Rhim, A. J. Freeman,J. Chem. Phys., 2013, 138, 164503.

[69] Y. J. Kim, J. Mater. Sci., 2018, 53, 10513.

[70] M. L. Chabinyc, M. F. Toney, R. J. Kline, I. McCulloch, M. Heeney, J. Am. Chem. Soc. 2007, 129, 3226.

[71] T. Lei, J.-H. Dou, J. Pei, Adv. Mater. 2012, 24, 6457.

[72] T. Olla, O. A. Ibraikulov, S. Ferry, O. Boyron, S. Méry, B. Heinrich, T. Heiser, P. Lévêque, N. Leclerc, Macromolecules, 2019, 52, 8006.

[73] X. Zhao, G. Xue, G. Qu, V. Singhania, Y. Zhao, K. Butrouna, A. Gumyusenge, Y. Diao, K. R. Graham, H. Li, J. Mei, Macromolecules 2017, 50, 6702. 


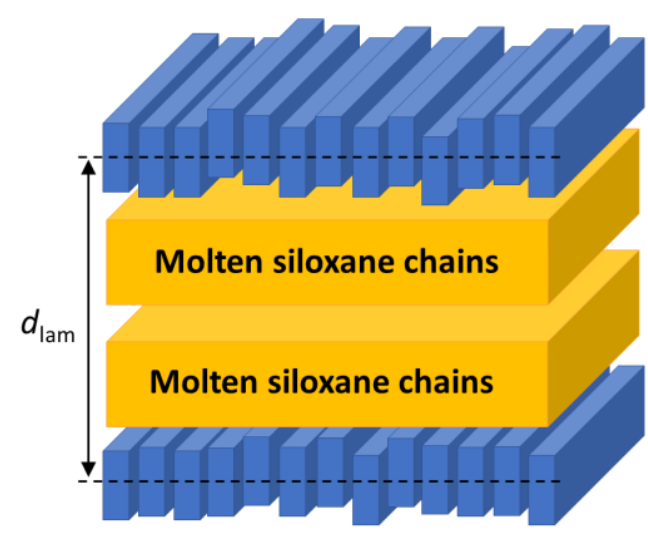

\section{Segregation + fluidity of siloxanes}

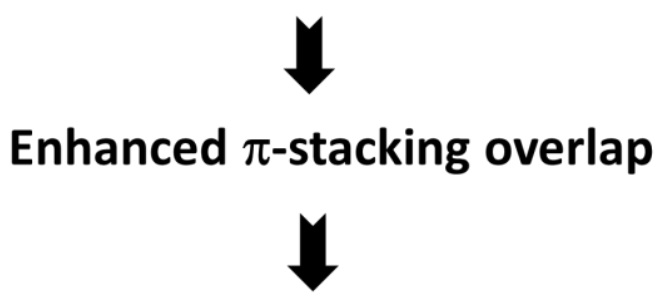

Higher mobility

The origin of the enhanced charge transport properties in siloxane-functionalized $\pi$ conjugated polymers was demonstrated. By scrutinizing the fine structural organization induced by linear siloxane and ramified alkyl side-chains of same molecular section, it was observed that the unique fluidity and segregation propensity of siloxane chains drove the siloxane-functionalized polymers to a considerable increase of the $\pi$-stacking backbone overlap. 